\title{
The Effect of Transcranial Direct Current Stimulation on Dorsolateral Prefrontal Cortex: a Review of its Role on Cognitive Functions
}

\author{
Bakhtiar Moslemi, Masoumeh Azmodeh*, Seyed Mahmoud Tabatabaei, Marzieh Alivandi Vafa
}

Department of Psychology, Tabriz Branch, Islamic Azad University, Tabriz, Iran

Article Info:

\section{A BSTRACT}

Introduction: The dorsolateral prefrontal cortex (DLPFC) is often selected as an excitatory target in the brain. A wide range of cognitive functions that can be modulated by the stimulation of this area makes it difficult to predict precise stimulation effect. The stimulation of DLPFC by transcranial direct current stimulation (tDCS) can be effective in modifying a wide range of cognitive activities. From a relatively simple and low-level activity, such as the attention process, to the complex cognitive functions, such as decision making and working memory, can be affected by tDCS. The effects of tDCS are very diverse and dependent on stimulation parameters, which sometimes lead to conflicting results. The aim of the present study was to investigate and identify various cognitive domains resulting from the effect of tDCS on DLPFC. Conclusion: Several complex cognitive activities are carried out by the DLPFC. The s timulation of this area by tDCS simultaneously activates multiple cognitive processes. This can lead to the facilitatation and sometimes inhibition of cognitive function, which subsequently may promote some behavior dysfunctions. To improve our understanding of the neurobiological mechanisms of tDCS on cognitive functions modulated by DPFLC, further investigations are needed.

\section{Key words:}

1. Transcranial Direct

Current Stimulation

2. Cognition

3. Brain

*Corresponding Author: Masoumeh Azmodeh

E-mail:Mas_azmod@yahoo.com 


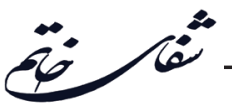

\title{
تأثير تحريك الكتريكى مغزى بر ناحيئ پيش ييشانى پشتنى -جانبى: مرورى بر نقش آن در عملكردهاى شناختى
}

\author{
بختيار مسلمى'، معصومه آزموده"، سيدمحمود طباطبايى، مرضيه عليوندى وفا \\ كَروه روانشناسى، واحد تبريز، دانشَاه آزاد اسلامى، تبريز، ايران
}

\section{اطلاعات مقاله:}

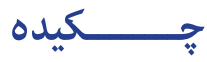

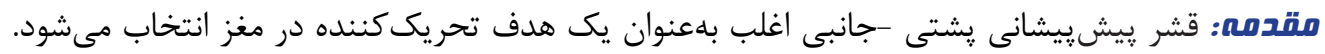

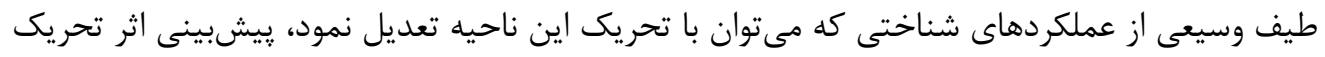

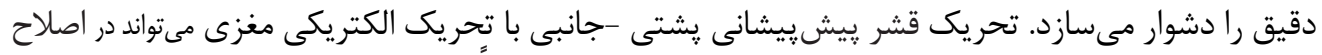

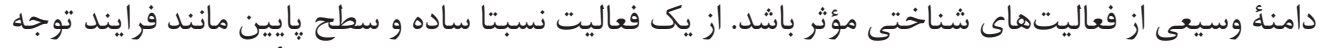

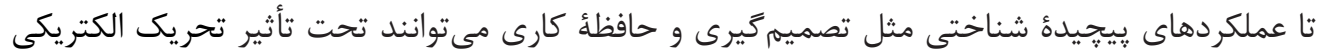

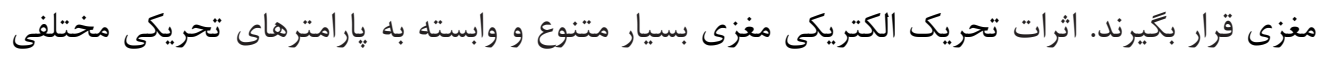

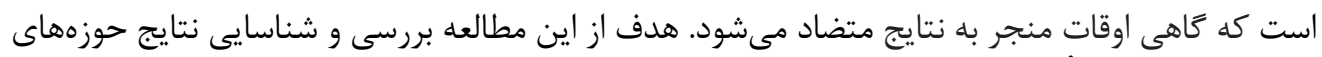

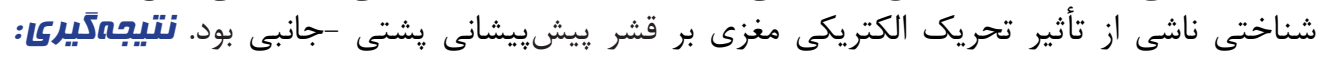

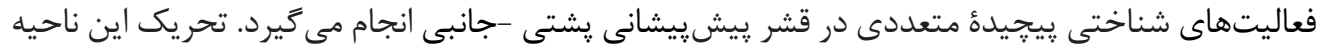

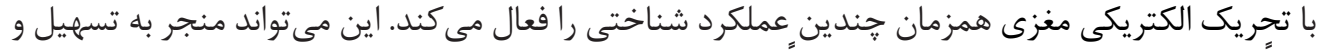

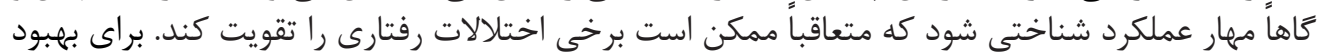

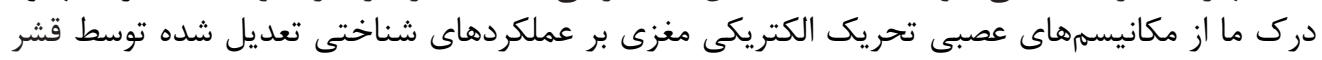

$$
\text { يِيشيشانى يشتى -جانبى، تحقيقات بيشترى مورد نياز است }
$$

كليد وازمها:

: م:

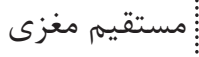

r.

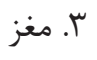

" نويسنده مسئول: معصومه آزموده

آدرس الكترونيكى: Mas_azmod@yahoo.com 


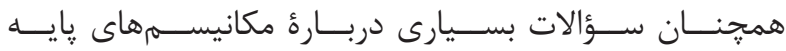

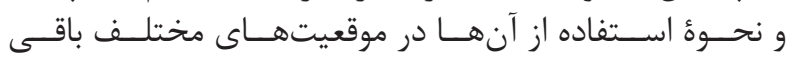

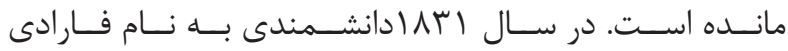

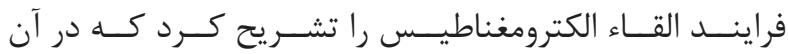

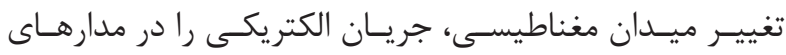

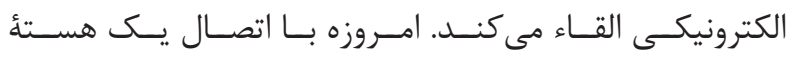

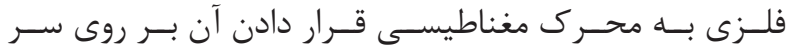

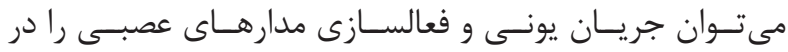

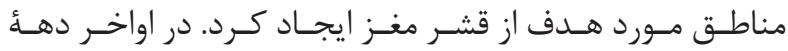

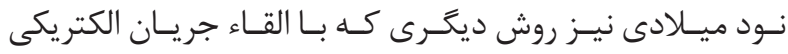

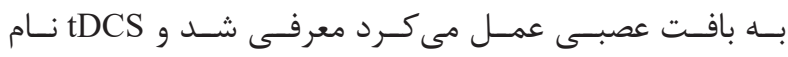

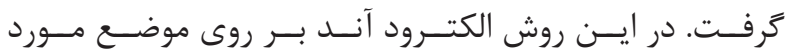

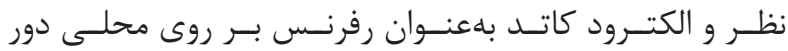

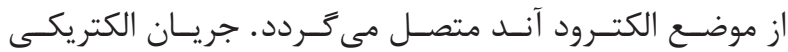

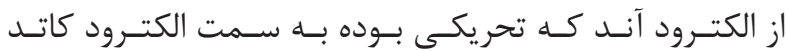

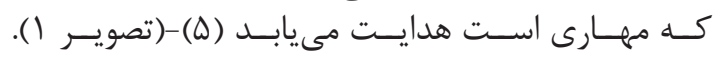

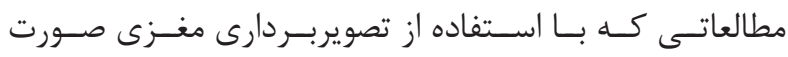

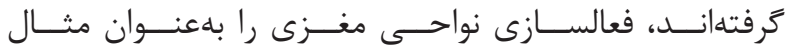

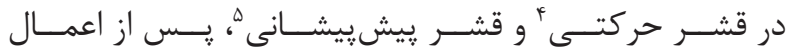

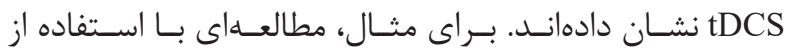

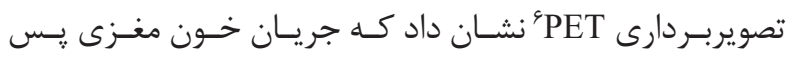

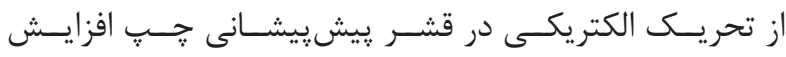

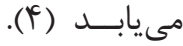

و و مكانيسم عملكرد آن

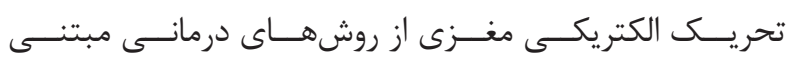

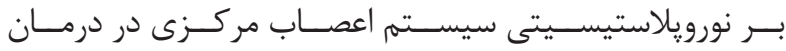

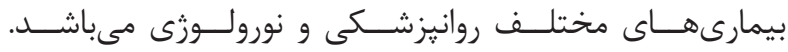

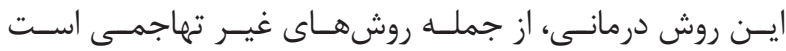

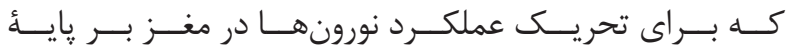

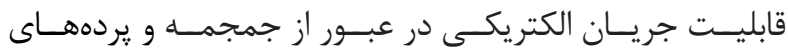

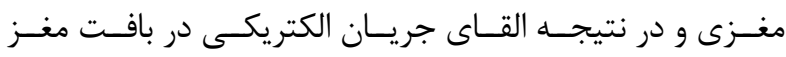

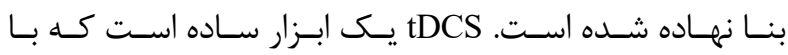

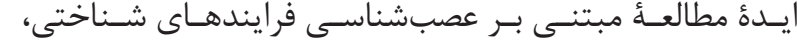

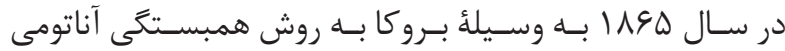

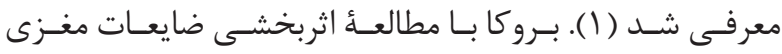

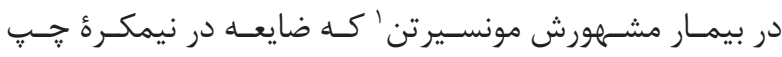

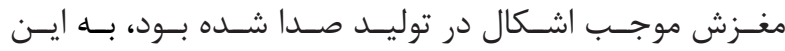

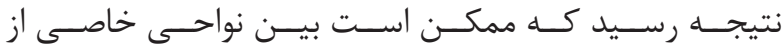

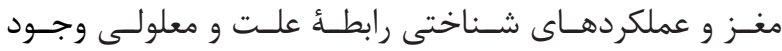

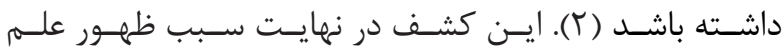

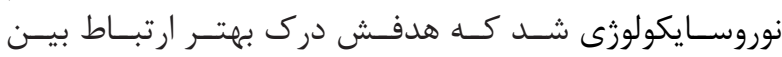

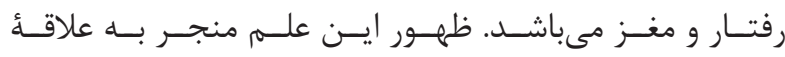

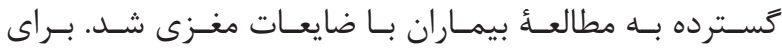

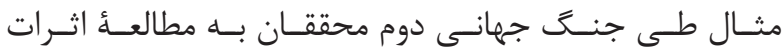

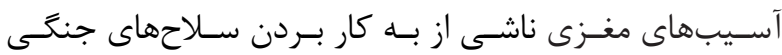

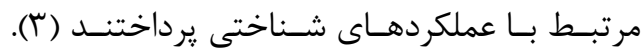

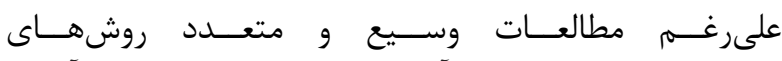

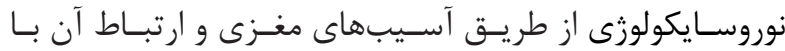

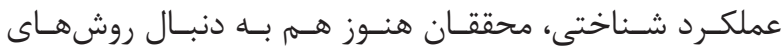

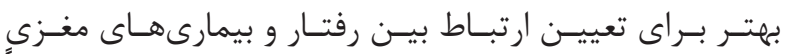

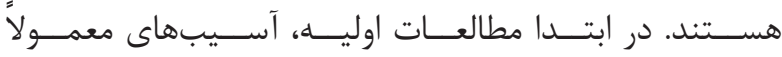

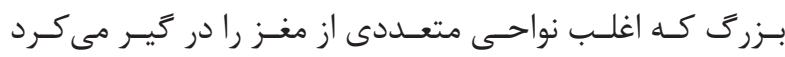

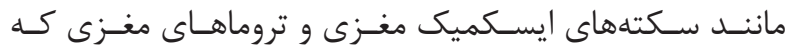

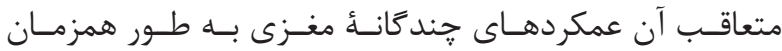

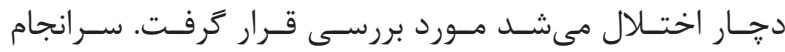

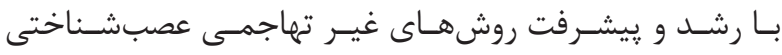

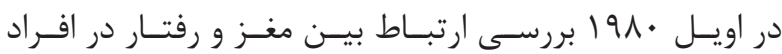

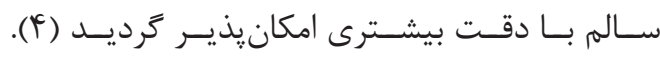

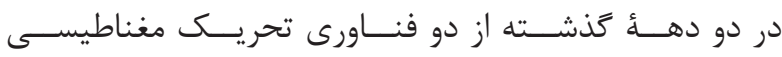

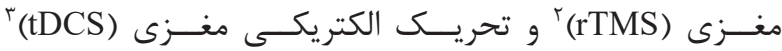

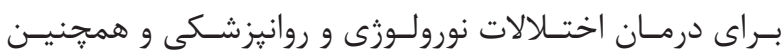

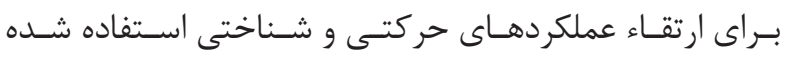

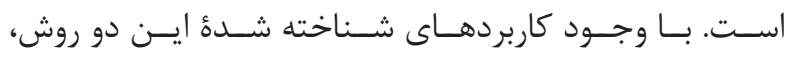

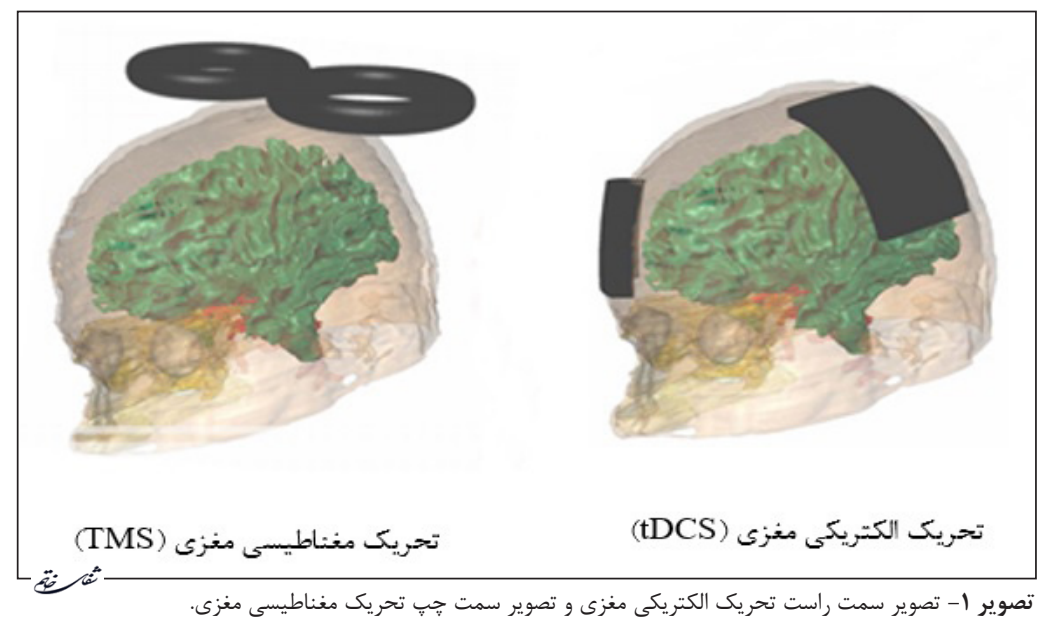

${ }^{1}$ Monsieur tan

${ }^{2}$ Transcranial direct current stimulation

${ }^{3}$ Transcranial magnetic stimulation

\footnotetext{
${ }^{4}$ Motor cortex

${ }^{5}$ Prefrontal cortex

${ }^{6}$ Positron emission tomography
} 
هـم در اثـرات اصلاحـى و هــم در انتخــاب يارامترهـاى tDCS

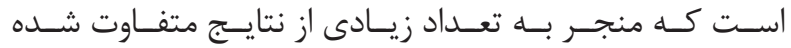

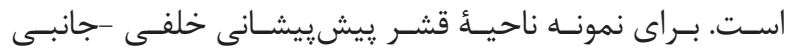

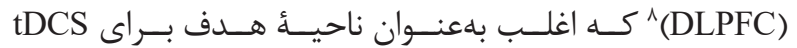

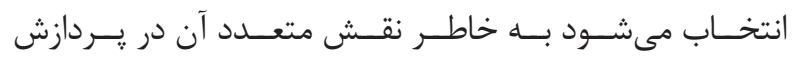

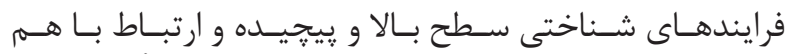

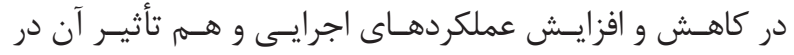

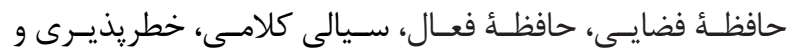

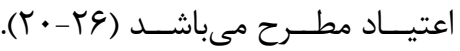

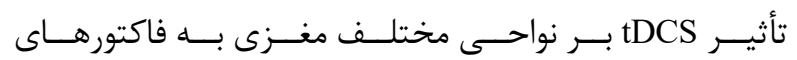

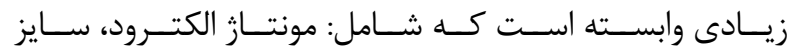

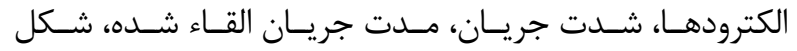

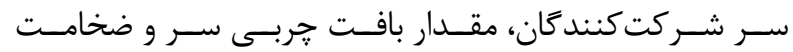

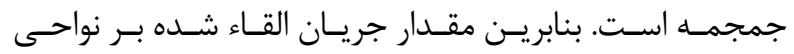

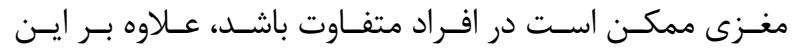

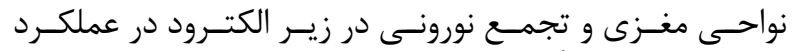

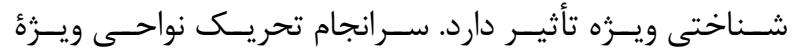

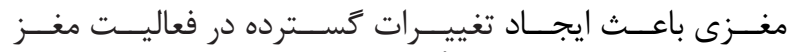

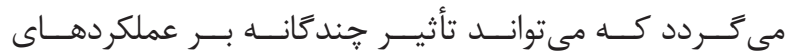

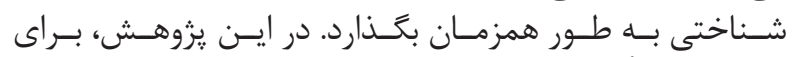

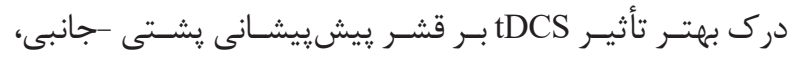

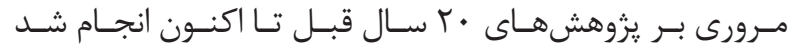

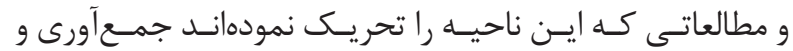

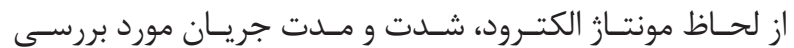

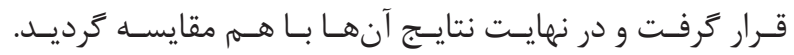

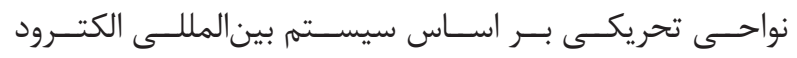

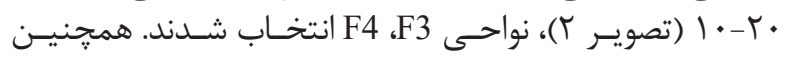

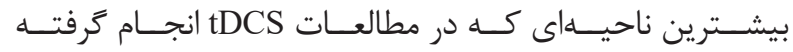

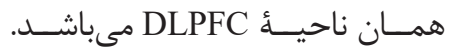

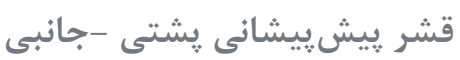

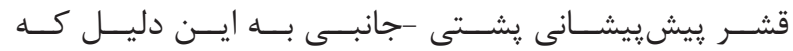

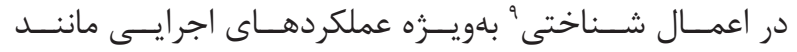

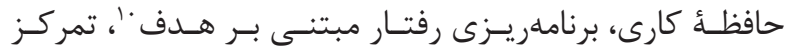

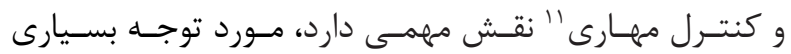

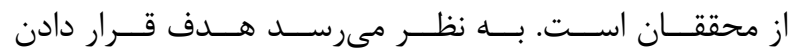

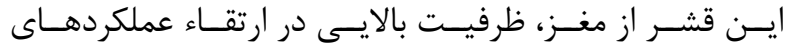

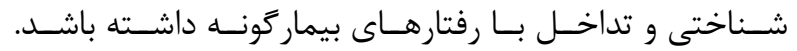

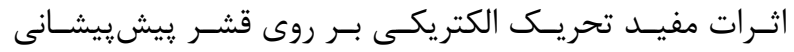

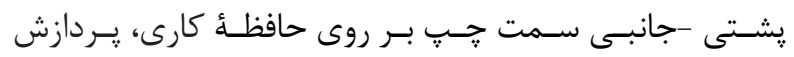

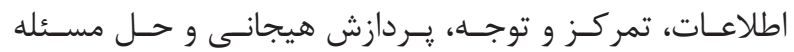

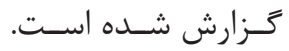

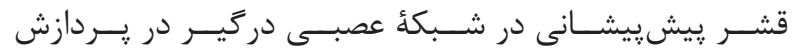

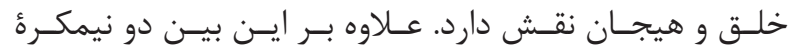

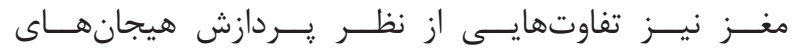

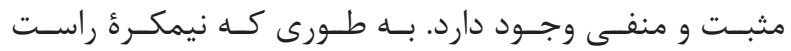

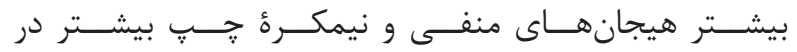
${ }^{7} \mathrm{rCBF}$

${ }^{8}$ Dorsolateral prefrontal cortex

${ }^{9}$ Cognitive functions
اسـتفاده از الكترودهــاى نسـبتاً بزرخى كـهـ روى سـر فـرد قـرار

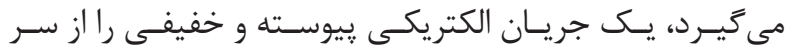

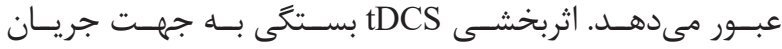

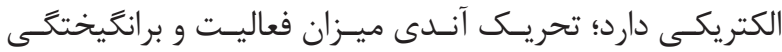

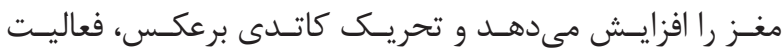

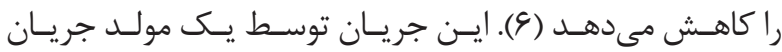

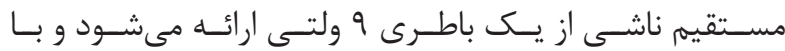

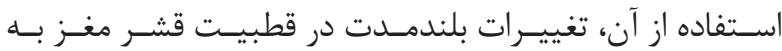

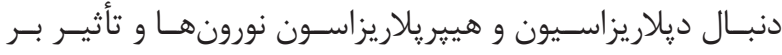

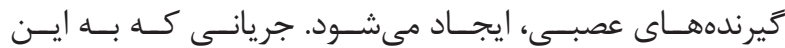

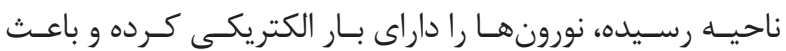

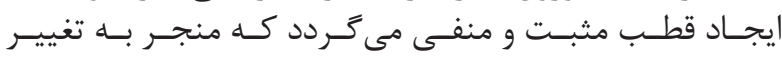

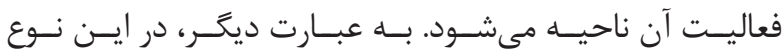

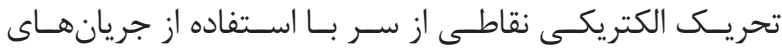

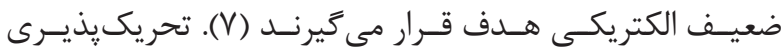

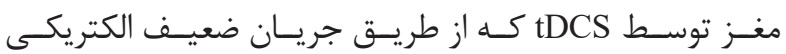

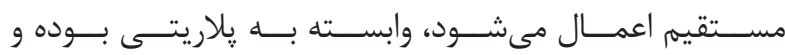

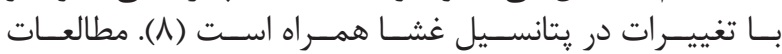

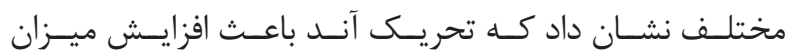

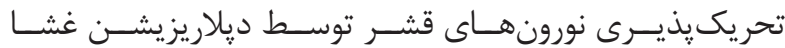

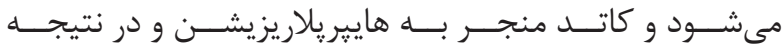

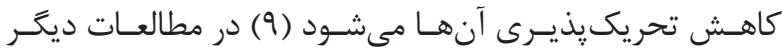

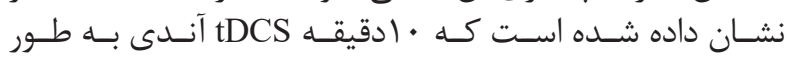

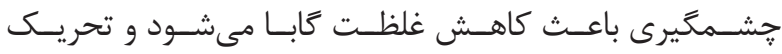

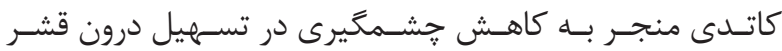

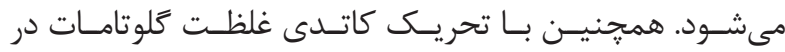

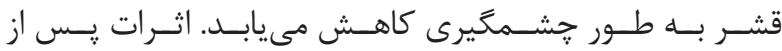

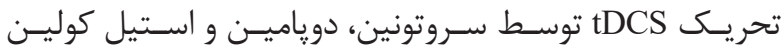

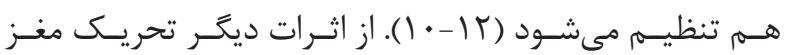

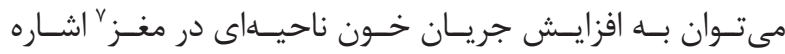

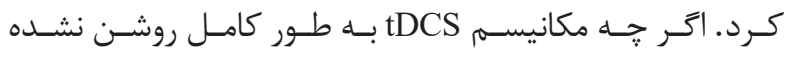

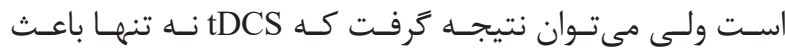

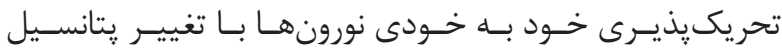

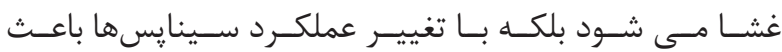

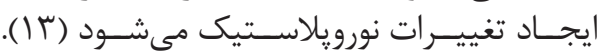

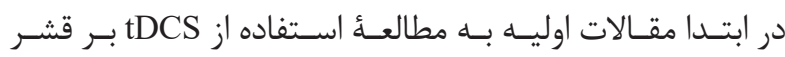

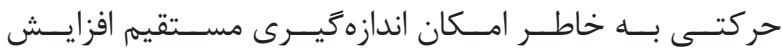

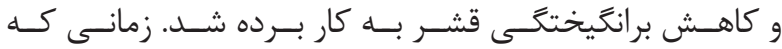

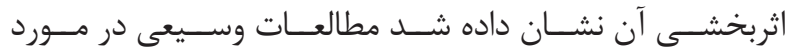

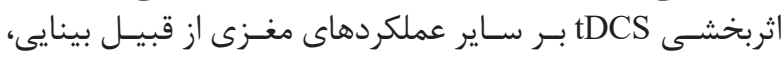

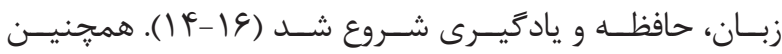

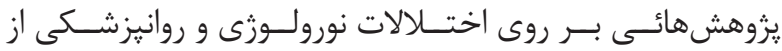

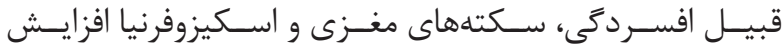

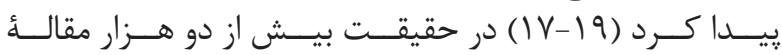

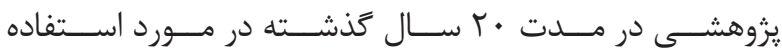

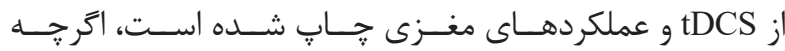

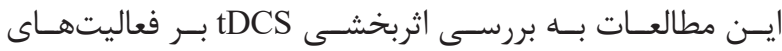

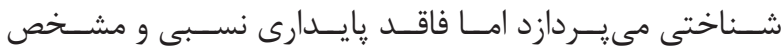

${ }^{10}$ Goal-directed behavior

${ }^{11}$ Inhibitory control 


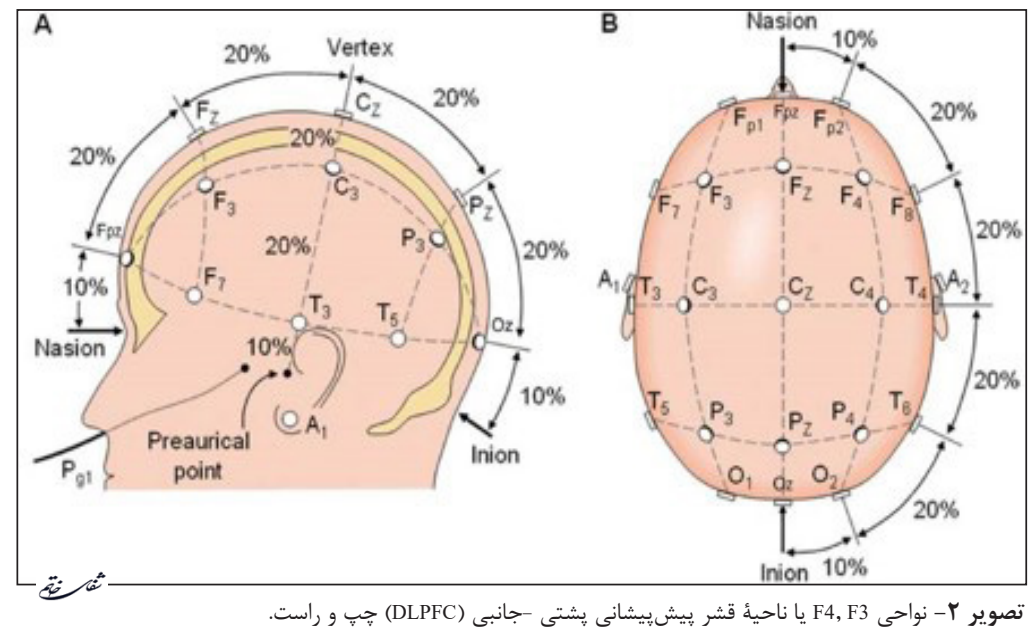

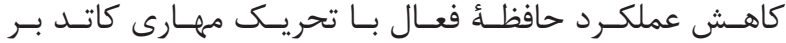
ناحيـهُ جــ هـPFC

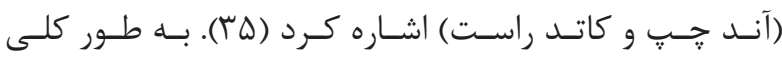

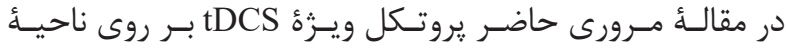

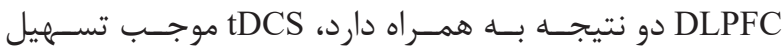

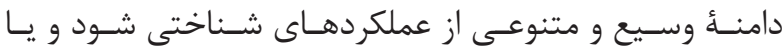

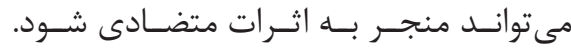

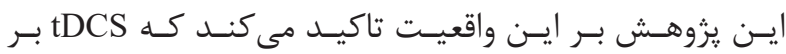

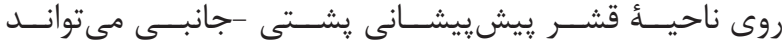

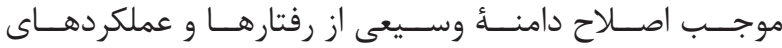

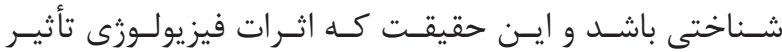

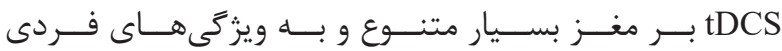

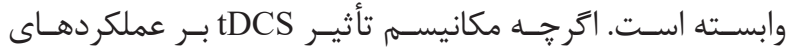

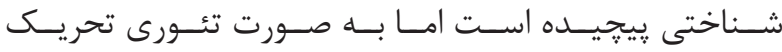

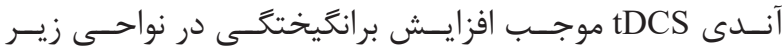

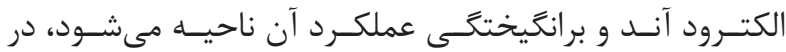

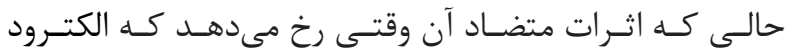

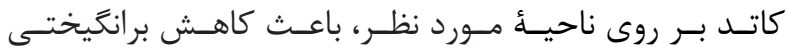

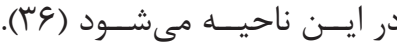
قطبيت نيذيرى

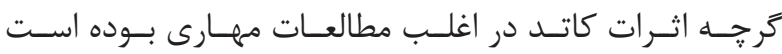

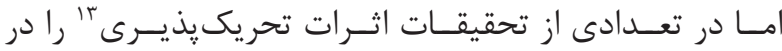

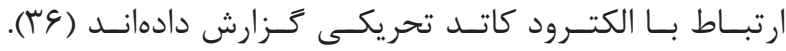

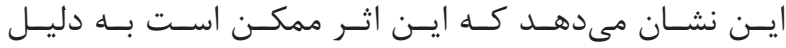

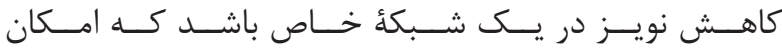

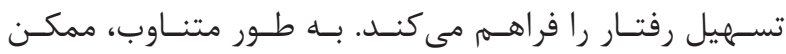

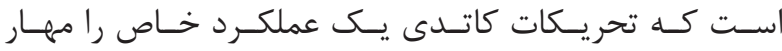

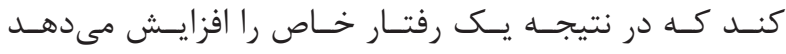

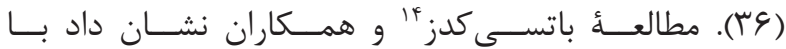

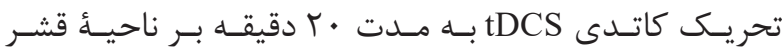

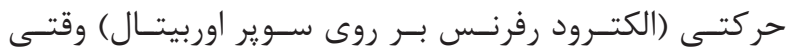

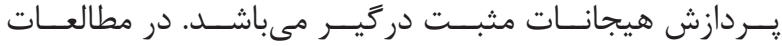

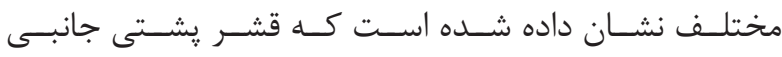

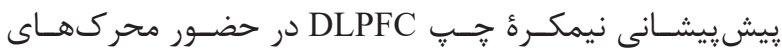

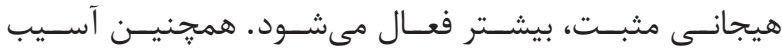
DLPFC

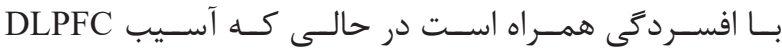

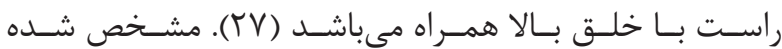

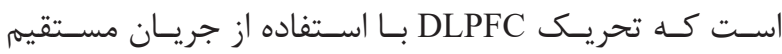

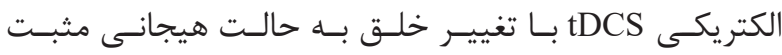

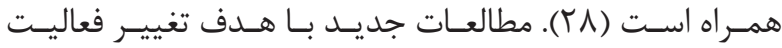

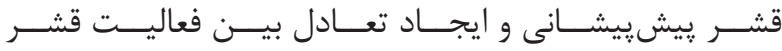

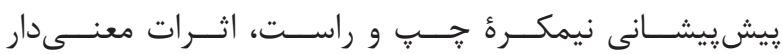

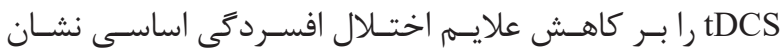

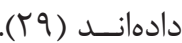

تعـدادى از مطالعـات، تأثيــات الكتريكـى مغزى را بــر جنبههاى

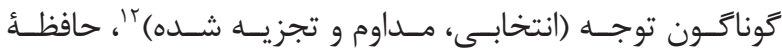

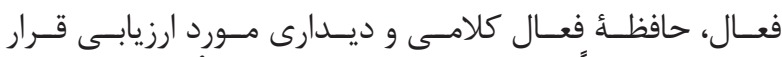

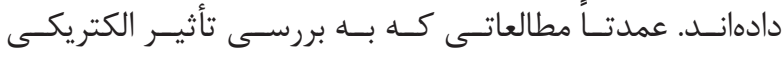

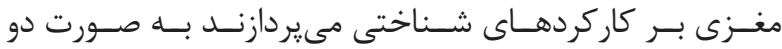

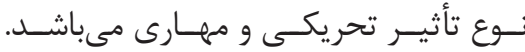

نتيجه گَيرى

بــه طـور خلاصـه tDCS بـا تحريـك DLPFC مى توانـد طيـف

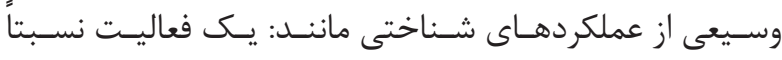

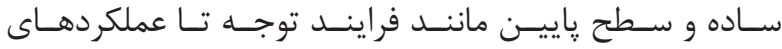

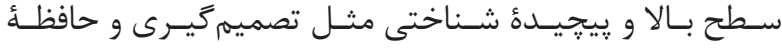

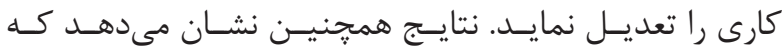

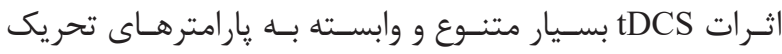

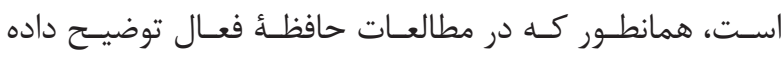

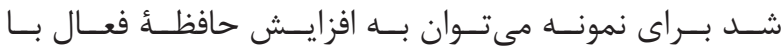

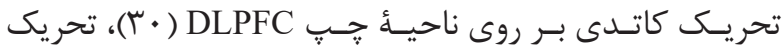

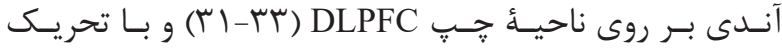

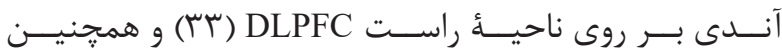

${ }^{12}$ Attention (selective, sustained, divided)

${ }^{13}$ Polarity

${ }^{14}$ Batsikadze 


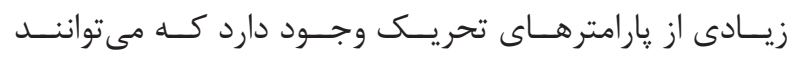

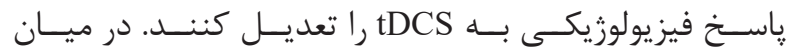

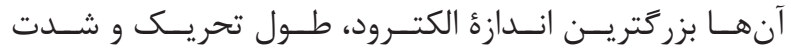

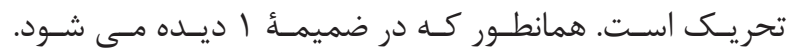

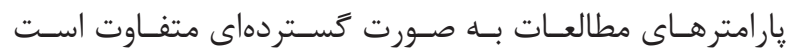

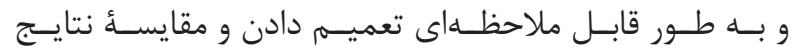

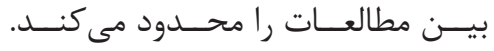

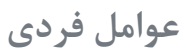

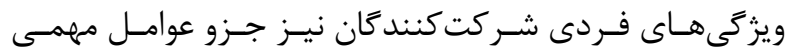

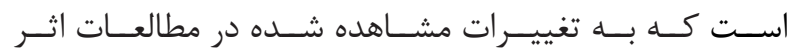

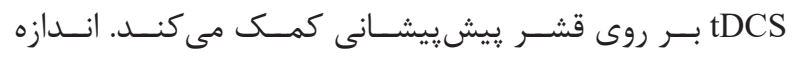

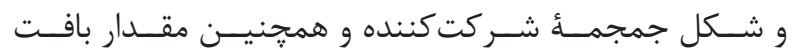

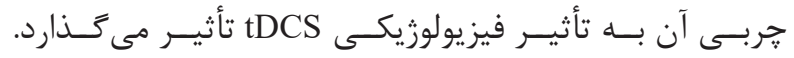

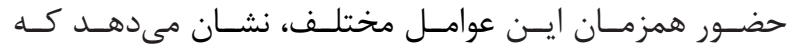

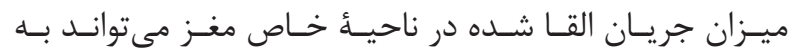

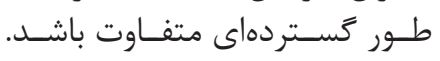

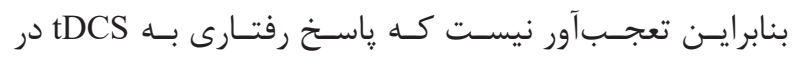

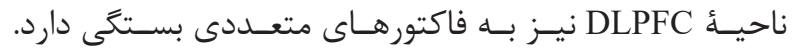

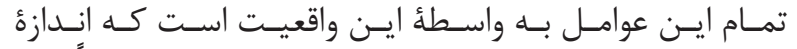

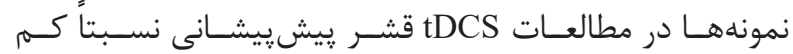

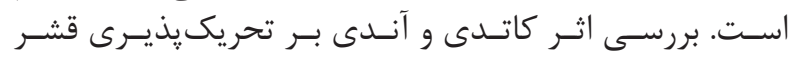

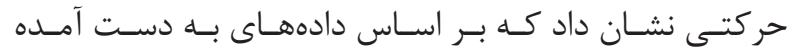

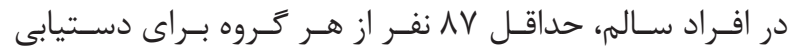

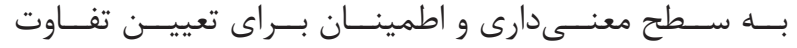

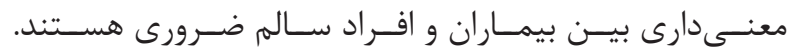

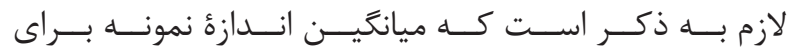

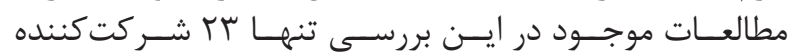

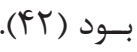

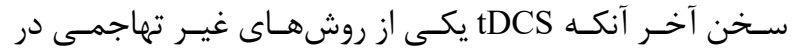

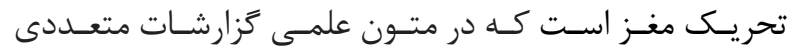

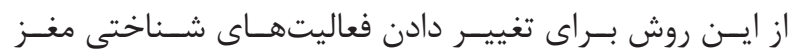

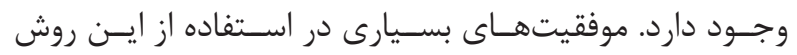

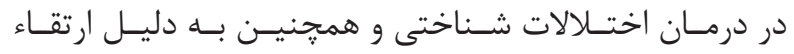

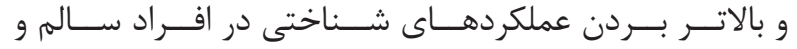

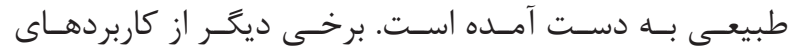

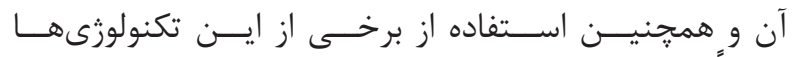

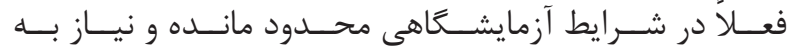

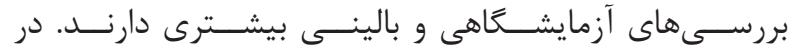

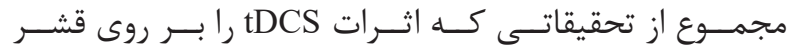

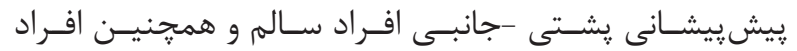

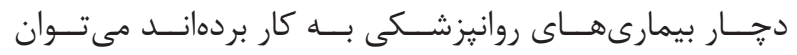

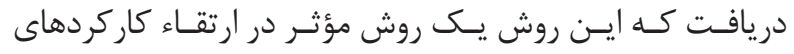

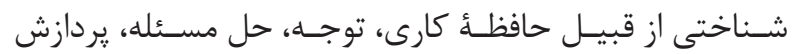

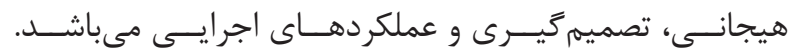

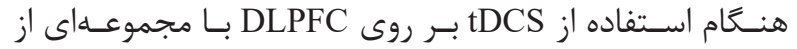

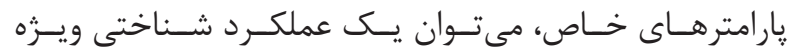

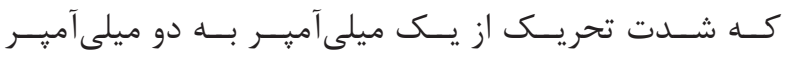

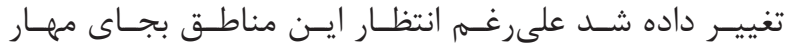

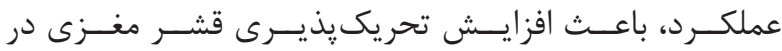

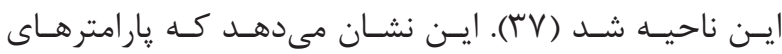

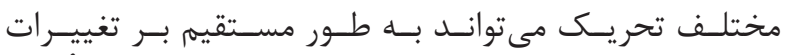

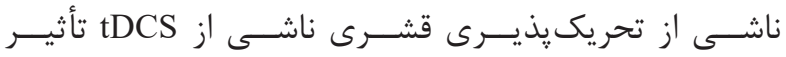

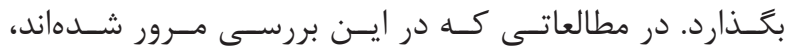

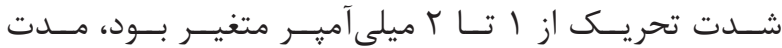

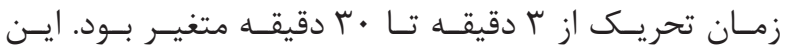

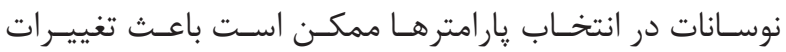

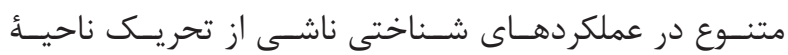

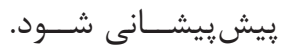
اصل وابسته به حالت

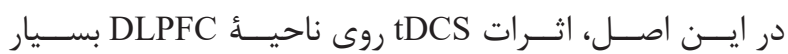

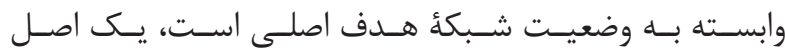

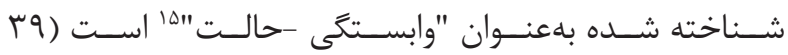

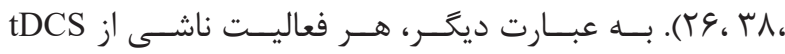

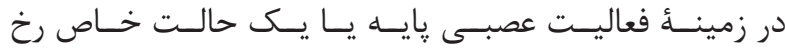

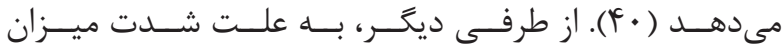

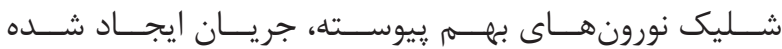

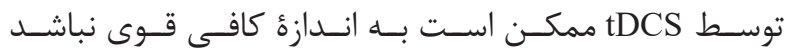

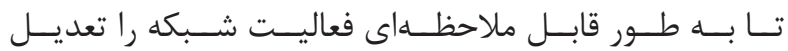

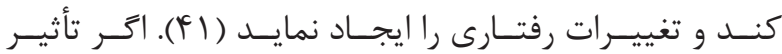

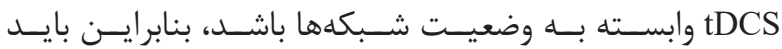

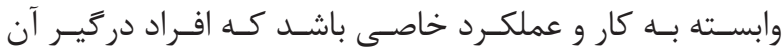

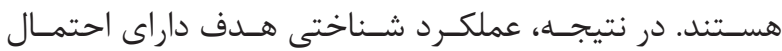

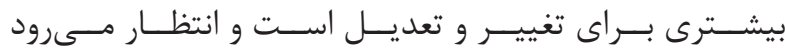

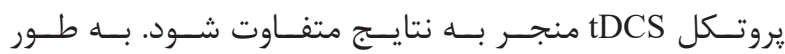

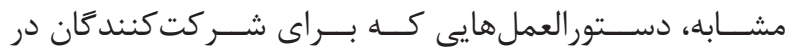

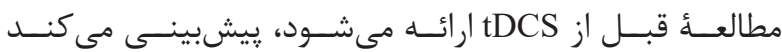

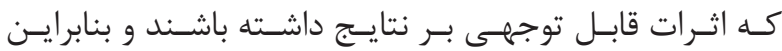

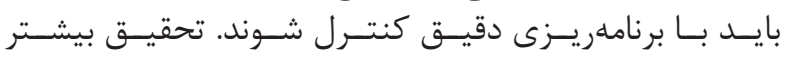

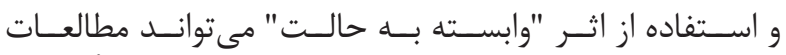

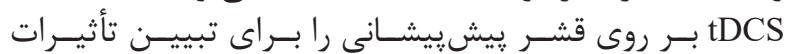

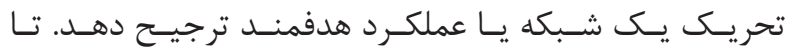

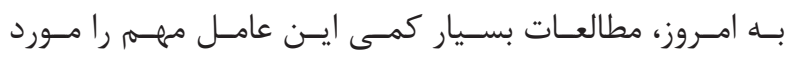

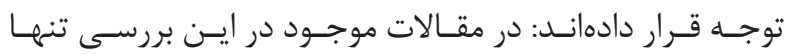

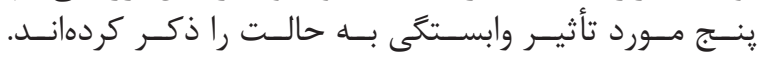

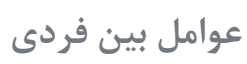

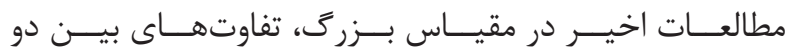

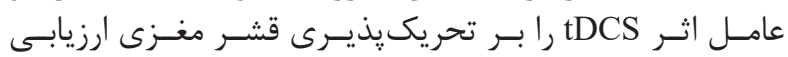

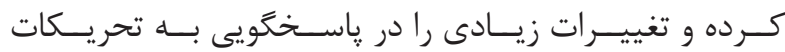

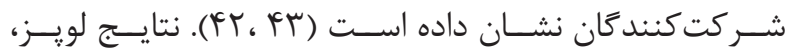

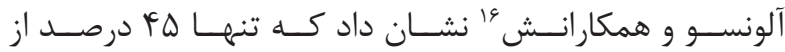
شــركت كنند

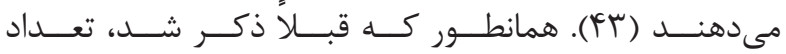




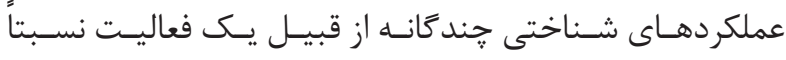

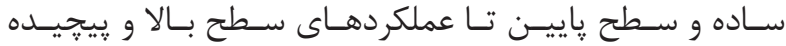

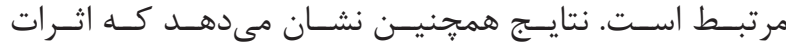

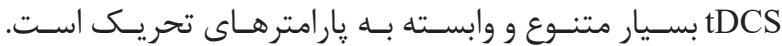

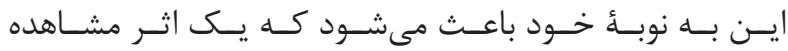

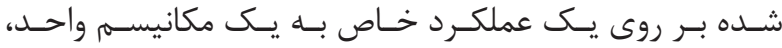

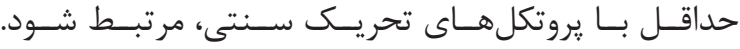

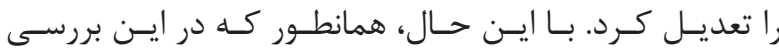

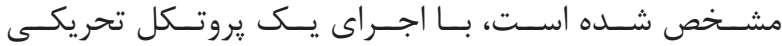

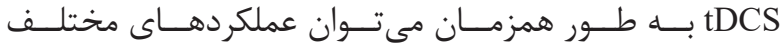

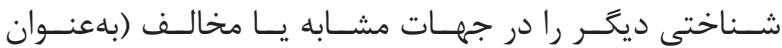

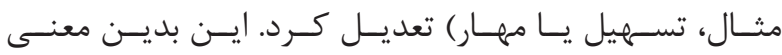

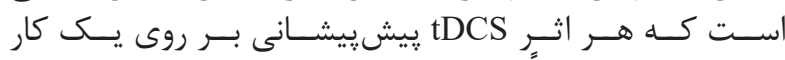

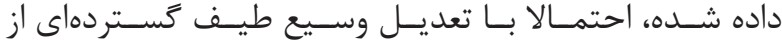

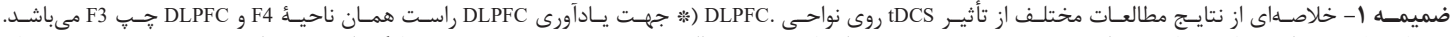

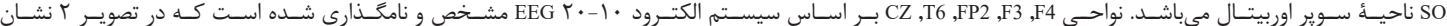

\begin{tabular}{|c|c|c|c|c|c|c|}
\hline نتايج & شله جريان & تصريك & عونتارُ & ناميأ مساف & هاف & ئزوهشكر \\
\hline اقزايش عملكرد & ا ميلى آمير & 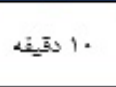 & 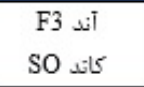 & جDLPFC & ماتظة كارى & قرثنى و همكاران ( \\
\hline اقزايش عملكرد & $\begin{array}{l}\text { ميكروآمير } \\
\end{array}$ & انديقه & 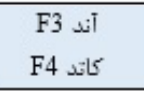 & 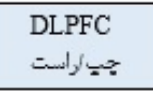 & ماتظد كارى & 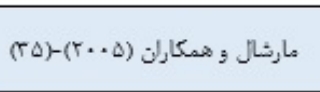 \\
\hline كاهش هيجانات منفى در اقراد & ا ميلى آمير & r آتيقة & 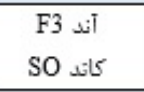 & جـ DLPFC & يرنازش & 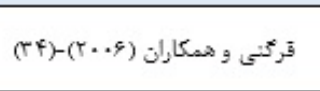 \\
\hline هوثر ير ريسكيذيرى & r بيلى آمير & . & 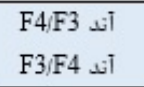 & 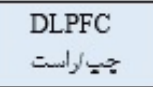 & نيسكيذيرى & 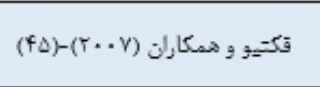 \\
\hline اقزايش عملكرد & r بيلى آمير & . & 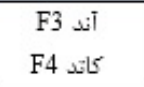 & 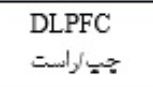 & ولى غتايع. & 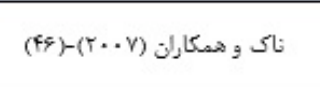 \\
\hline اقزايش عملكرد & ميلي/آهير & أ إتيقد & 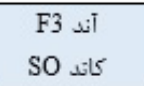 & $\begin{array}{l}\text { DLPFC } \\
\text { ?ב fاست }\end{array}$ & تصميمئكيرى & يريور و همكاران (TV)-(TV) \\
\hline كاهش رقتارهاى ريسكيذيرى & ا ميلىآمير & 10 & 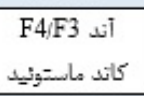 & 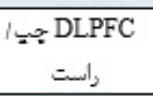 & نيسكيذيرى & ييلى و ممكاران ( \\
\hline ئبيود عملكرد & 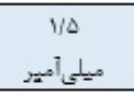 & ه/ه دقيقه & 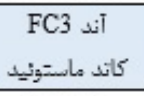 & DLPFC & عملكرد اجمرائى & 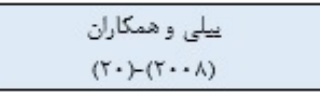 \\
\hline كاهش ادراك درد & r بيلى آمير & ه دقيقه & $\begin{array}{l}\text { F3 آند SO } \\
\text { SO }\end{array}$ & $\because \mathrm{DLPFC}$ & ادراك درد & 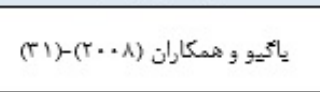 \\
\hline اقزايش عملكرد & ا ميلي آمير & . Tآيقدا. & 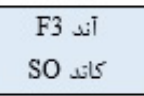 & $\leadsto$ DLPFC & ماتظة كارى كلاهى & 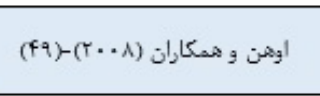 \\
\hline كاهش هيجانات منفى & r بيلى آمير & ه دقيقه & 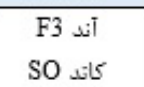 & $\approx$ DLPFC & يرنازثي & ياءيو و ممكاران (T)-(T) \\
\hline اقززايش حاقظل كارى & r بيلمىآمير & . & 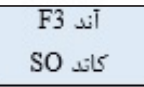 & $=$ DLPFC & ماتظلة كارى & جو جم و همكارلن (r.. \\
\hline أقزايش توجه نسيت يه كروه & r ميلمىآمير & . ب نقيفة & كاتد SO آند SO & $\approx$ DLPFC & توجيه & 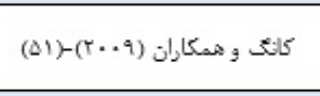 \\
\hline ي يجيود عملكرد & ا ميلى آمير & . T بقيفة & 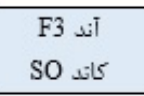 & $\approx$ DLPFC & حل مسنك & 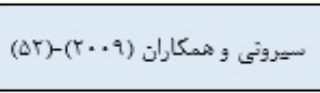 \\
\hline اقزايش عملكرد & ا ميلى آمير & اندية 10 & 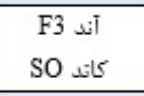 & $\cong$ DLPFC & 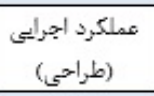 & دوكرى و همكاران (T) (T)-(T) \\
\hline 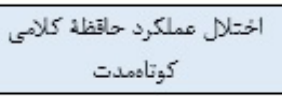 & ميلميآير & ه دقيقه & 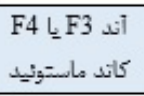 & 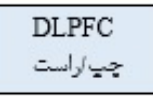 & 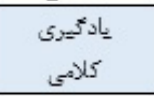 & 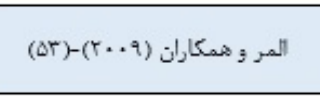 \\
\hline تسهيل عملكرد & r بيلىى آمير & ا آقيفة & 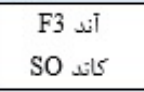 & $\simeq$ DLPFC & (السمبريشيى) & 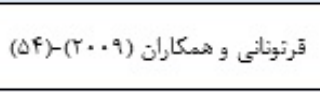 \\
\hline يجيون عملكرد & r ميلمىآمير & با نديقة & $\begin{array}{l}\text { F4/F3 كاتد آند F3/F4 } \\
\text { F34 }\end{array}$ & 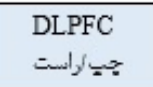 & تصميمكيرى & هيج و همكارلن (·- (r. \\
\hline ي يجيود عملكرد & r بيلمىآمير & إنيقه 10 & $\begin{array}{c}\text { F4/F3 كاتد نلتوئيد } \\
\text { آند راست } \\
\end{array}$ & $\begin{array}{l}\text { DLPFC } \\
\text { צبs اسات }\end{array}$ & تصميمثكيرى & 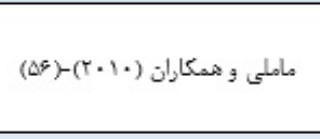 \\
\hline يجيود تصميمثكيرى & ب إيلى آمير & ا إتيقه & $\begin{array}{l}\text { F4/F3 آند كاتد F3/F4 } \\
\text { F34 }\end{array}$ & $\approx$ DLPFC & تصميمثكيرى & 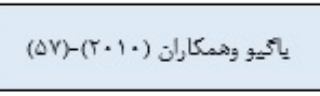 \\
\hline كامش عملكرد & ا ميلمىآمير & ا التيفة & $\begin{array}{l}\text { آند ك4/F3 كاتد CZ } \\
\text { كاتد }\end{array}$ & $\begin{array}{l}\text { DLPFC } \\
\text { جب:yاست }\end{array}$ & طيقعيندى ئيرى & 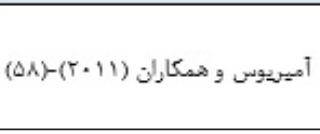 \\
\hline
\end{tabular}




\begin{tabular}{|c|c|c|c|c|c|c|}
\hline اقزايش عملكرد & ا ميلى آمير & ال آقيفه & 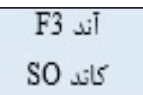 & جي DLPFC & ماتظلة كارى & 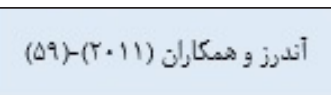 \\
\hline يردازش كند هيجانات هنفى و & ا ميلى آمير & 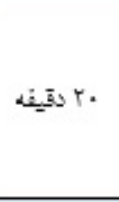 & 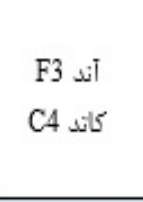 & مي DLPFC & تنظيم ميجانى & 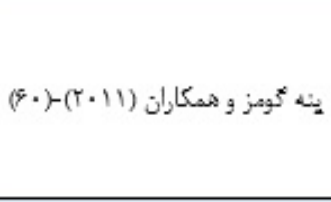 \\
\hline اقزايش عملكرد & ا ميلمىآمير & 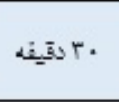 & كاتد & جي DLPFC & ماتظكه & 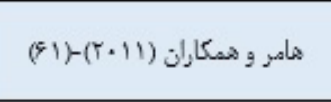 \\
\hline اقزايش عملكرد & ا ميلى آمير & ان آتيفه & 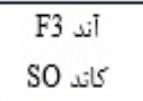 & مي DLPFC & عملكرد اجرايى & ليت و همكارلن (II (T)-(ET) \\
\hline اقزايش عملكرد & ا ميلى آمير & ال آتيقها. & 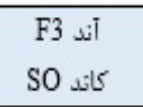 & $\approx$ DLPFC & ماتظف كارى & 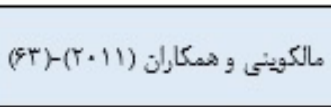 \\
\hline اقزايش تنظيم هيجاتى & ا ميليلى آمير & . & 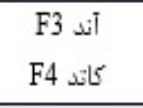 & جي DLPFC & يردازش & 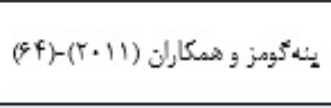 \\
\hline اقززايش عملكرد & ا ميلى آمير & r. ثليقه r. & 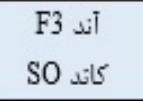 & جي DLPFC & ماتظة كارى & تثو و همكاران (T) (T)- (T) \\
\hline كاتد: كاهش عملكرد آند عملكرد & ا ميلمىآمير & انقيفة & 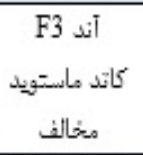 & جي DLPFC & ماتظة كارى & 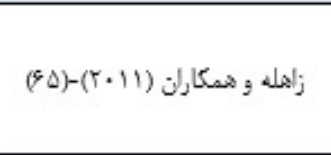 \\
\hline اقزايش ياسغماى مطمثن و قورىى & r بيلىآمير & . & $\begin{array}{l}\text { F4/F3 كاتد F3/F4 } \\
\text { F3/ }\end{array}$ & جDLPFC & تصميمكيرى & ميناتى و ممكاران (T/ · (T)-(89) \\
\hline اقززايش عملكرد & r ميلى آمير & انديفة & 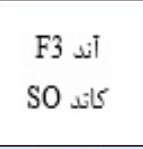 & جي DLPFC & يكيارجكيى يرنى & يالكونى و ويتالونى (T) • (T)-(FV) \\
\hline اقزايش عملكرد & ا ميلميآمير & ا ناقيفه & 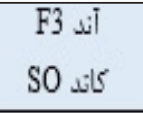 & $\approx$ DLPFC & ماتفلة كارى & 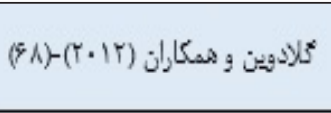 \\
\hline اقزايش عملكرد & با ميلى أمير & ان & 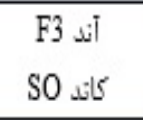 & $\approx$ DLPFC & تنظيم ثيجانى & 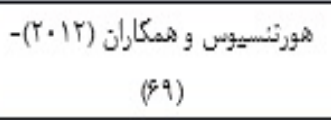 \\
\hline كاتند: آقزايش عملكرد ماتظله & كيأمير & . تآيفر. & 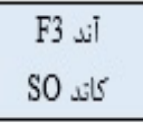 & $\approx$ DLPFC & ماتظلة آشكار & جوادى و ولش (rاء (r)-(r) \\
\hline اقزايش عملكرد ماقظله & ميلمي آمير & r بقيفر & 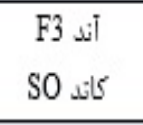 & هي DLPFC & ماقظة كالامى & جوادى و ومكاران (T/ (T/)-(V) \\
\hline اقزايش عملكرد محاقظه & ئأمير & . & 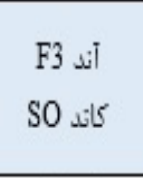 & $\begin{array}{l}\text { DLPFC } \\
\text { צب/אاست }\end{array}$ & تواقظة قعال، & جون و مان (TI) (TI (T) \\
\hline اقزايش عملكرد & ب الميلي|آمير & . & 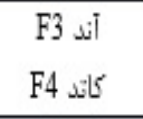 & 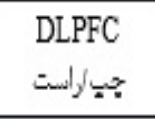 & عملكرد اجرايى & 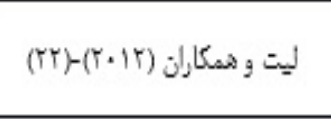 \\
\hline كاهش هيجانات منفى & اميليىآمير & 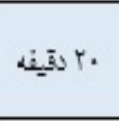 & 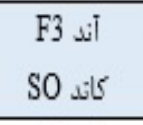 & $\approx$ DLPFC & $\begin{array}{l}\text { انعطاقيذيرى } \\
\end{array}$ & مايوكا و ممكاران (TT)-(TT) \\
\hline اقززايش عملكرد & اميلي|آمير & || آتيفه & 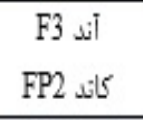 & ב DLPFC & يريازن & تتيزكى و فمكارلن (r) \\
\hline إقزايش نحمل نسيت يه درد & آ ميلي آمير & r بقيفه & 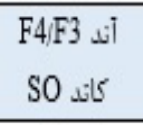 & 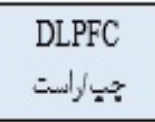 & عملكرد اجرايى & ميليوس و فمكاران (TF)-(T) \\
\hline اقززيش عملكرد & ب إيلمى آمير & 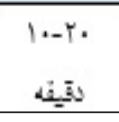 & 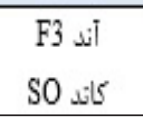 & حي DLPFC & ماقظلة كارى و & نيجه و همكاران (T (T) \\
\hline
\end{tabular}




\begin{tabular}{|c|c|c|c|c|c|c|}
\hline اقزايش عملكرد & ميلي آمير & إقيفة & 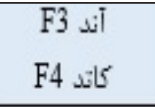 & $\begin{array}{l}\text { DLPFC } \\
\text { צב لyاست }\end{array}$ & يردازش & 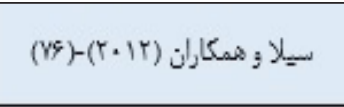 \\
\hline اقززايش عملكرد & ب آ ميلى آمير & 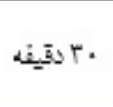 & 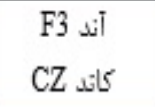 & هي DLPFC & ادراك نيان & وانتورسال و همكارلن (T) • (Tf)-(Tf) \\
\hline كامش تصميه كيرى ناسازتار & ميلىآهير & ا آقيةج & 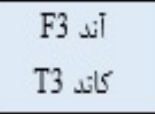 & هLPFC & 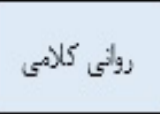 & 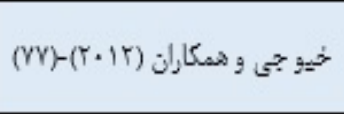 \\
\hline كاهش تصميمات خطرناك & ميلمآهير & الميفه & $\begin{array}{l}\text { F4/F3 كاتد F3/F4 } \\
\text { F3/Fد }\end{array}$ & $\begin{array}{l}\text { DLPFC } \\
\text { צב/لراست }\end{array}$ & تصميثمثيرى & يرييفل و ممكاران (T/ · (T/)-(V) \\
\hline اقززايش عملكرد & ب إيلى آمير & rا ثقيفه & 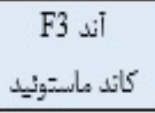 & مي DLPFC & نصميثمكيرى & آستنا و ممكاران (T/ • (Tr)(Tr) \\
\hline اقززايش نجرييات خوششايند & ب إميلى آمير & . & 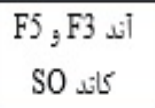 & هLDFC & ماقظلة ترس & 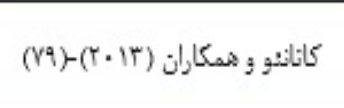 \\
\hline اقزايش عملكرد & ب آ ميلمآمير & . & 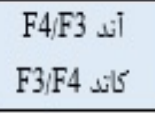 & $\begin{array}{l}\text { DLPFC } \\
\text { جב_/yاست }\end{array}$ & ميردازش & 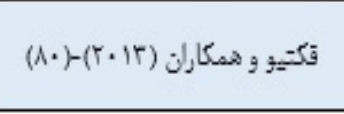 \\
\hline يجيود تصميهمثيرى & ميلى آهير & r. & 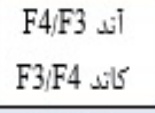 & هي DLPFC & تصميثمكيزى & هيج و همكاران (Tا • (T)-(A) \\
\hline اقزايش عملكرد & 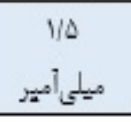 & . تقيف با. & 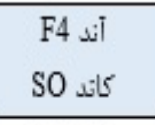 & راست DLPFC & تصميمثيرى يا تأخير & قيشر و كمكاران (TT)-(T) \\
\hline اقزايش يادكيرى حركتى & ب آميلىآمير & كا بقية & 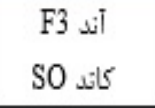 & حمLPFC & تنظيم ميجانى & قورستر و همكاران (T) (T) \\
\hline اقتزايش عملكرد & ميلي آهير & . & 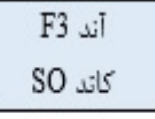 & בي DLPFC & يادكيرى & هوى و همكاران (Tf)-(T) \\
\hline اقزايش عملكرد ماقظه & ميلى آهير & . & 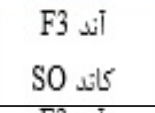 & هLPFC & ماتظد كارى & 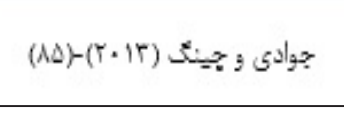 \\
\hline اقزايش عملكرد حاقظه & ميلي/آمير & . & كاتد S3 أند S3 & ج DLPFC & حاقظة كارى & 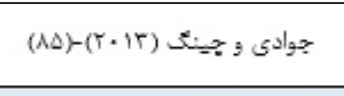 \\
\hline كاهش عملكرد و حذق اثر اوليه & ا ميلمىآمير & . & 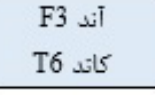 & $\approx$ DLPFC & يلندمدت & 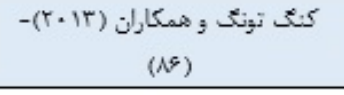 \\
\hline اقزايش عملكرث حاقظه در & ميلىآمير & F & 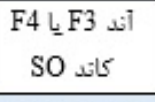 & $\begin{array}{l}\text { DLPFC } \\
\text { צب fاست }\end{array}$ & يردازش & 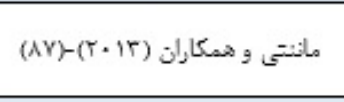 \\
\hline يجيزود عملكرد & ب ميلىآمير & 10 & 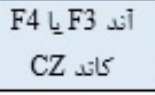 & $\begin{array}{l}\text { DLPFC } \\
\text { צب fاست }\end{array}$ & ماتظلة ديدارى & ميرون و لويدور (T) · (T) (M) \\
\hline ئبيون عملكرد & ب آميلميآمير & إتيقه & $\begin{array}{l}\text { F3/SO آند كاتد SO/F4 } \\
\end{array}$ & $\begin{array}{l}\text { DLPFC } \\
\text { جبكاست }\end{array}$ & ماتظة كارى & 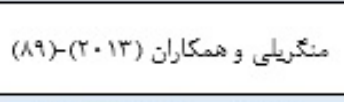 \\
\hline هوثر ير خلق & ا ميلى آهير & 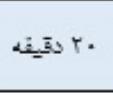 & 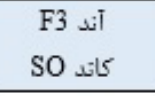 & جي DLPFC & تصميمكيرى & 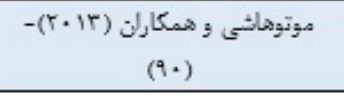 \\
\hline اقزايش عملكرد & ميليآمير & r. & 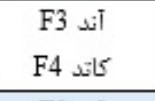 & $\begin{array}{l}\text { DLPFC } \\
\text { جبs fyاست }\end{array}$ & خحلق & 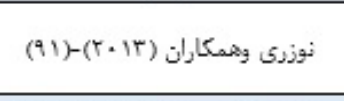 \\
\hline كاهش نشخوار ذهنى و اقَكار خود & r بيلى آمير & r. & 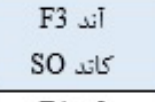 & جي DLPFC & توجه & واندرهاسيلت و همكاران (rا • (T)- \\
\hline ي يجيود عملكرد & ميلى|آهير & ا آقيقة & 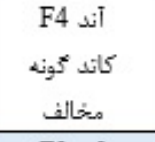 & راست DLPFC & 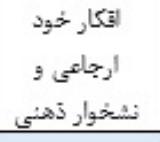 & تانو و همكاران (rا. (T)-(Tr) \\
\hline 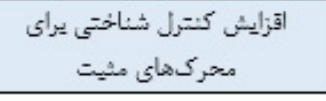 & r بيلى آمير & 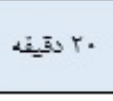 & 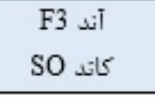 & جي DLPFC & توجه & 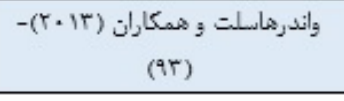 \\
\hline اعلاح عملكرد & ب آميلى آمير & 10 & 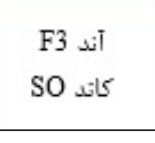 & جي DLPFC & ماقظة كارى و و يردازثل & 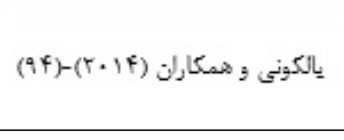 \\
\hline
\end{tabular}




\begin{tabular}{|c|c|c|c|c|c|c|}
\hline ئيود عملكرد & ا ميلميآمير & 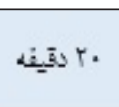 & 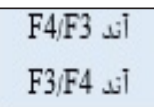 & $\begin{array}{l}\text { DLPFC } \\
\text { جب fاست }\end{array}$ & يردازش مـنايى & 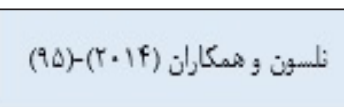 \\
\hline عدم نأثير ير يادكيرى ميتلى ير & ب الميلميآمير & ه ب تقيفه & $\begin{array}{c}\text { ك4 آند F4 كاتد } \\
\text { inion }\end{array}$ & راست DLPFC & نوجه & 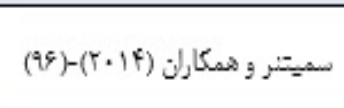 \\
\hline 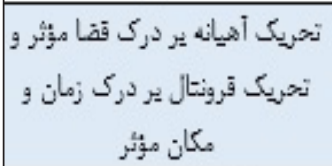 & ميلىآهير & 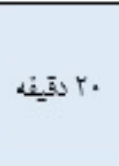 & 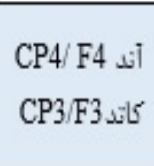 & راست DLPFC & 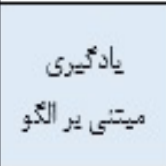 & 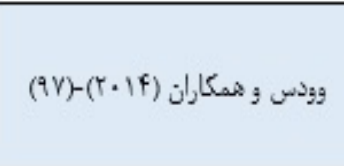 \\
\hline 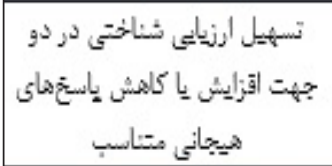 & r ميليى آمير & . & 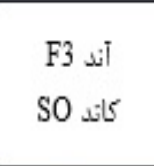 & جي DLPFC & درك مكان و & قيشر و همكاران (T)-(T) (9) \\
\hline 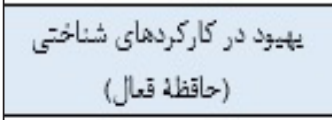 & r بيلي آمير & 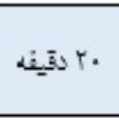 & 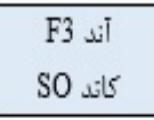 & $\cong \mathrm{DLPFC}$ & شنانتيتي & 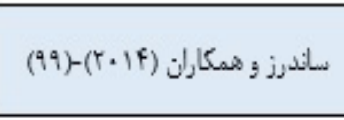 \\
\hline يجيود حاقظل كاري & ا ميليى آمير & r باتية r. & 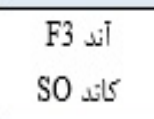 & جي DLPFC & كاركردماي & 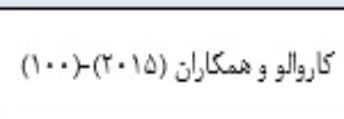 \\
\hline يجيون قضاوت انخاقى & ب الميلي آمير & ا القيفه & 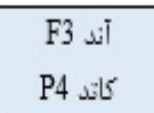 & هLPFC & ماتظة كارى & 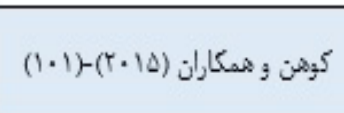 \\
\hline كايش اثرات منفى تصاوير منفىي & 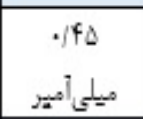 & ها نقيفه & 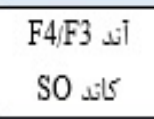 & 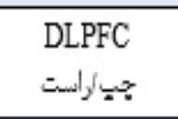 & قضاوت الخالقي & 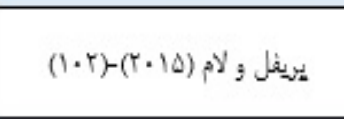 \\
\hline كاهش خطريذيرى & r ميليلى آمير & 19 انقيفة & 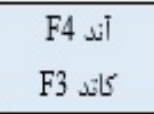 & راست DLPFC & خرودتنظيمى & جينت و لى (10) \\
\hline 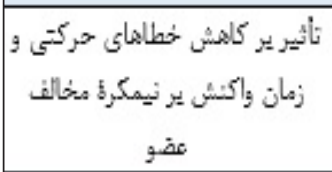 & r بيلى آمير & r بقية r. & 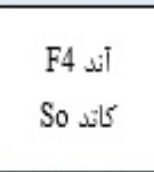 & راست DLPFC & تصميثمئيرى & ليدوس و همكارلن (10 +(t)-f(1) \\
\hline 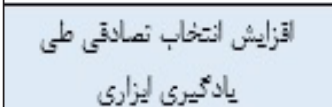 & ب آميلي آمير & ان آيفه & 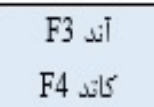 & راست DLPFC & عملكرد اندامهاما & 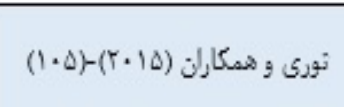 \\
\hline 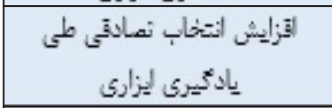 & ب الميلي|آمير & انيقية & 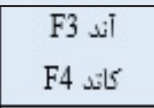 & راست DLPFC & عملكرد اندامهاما & 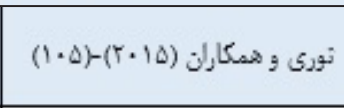 \\
\hline 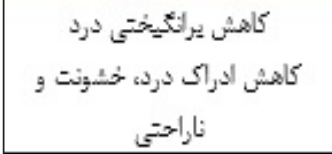 & ب الميليآمير & ها نقيفه & $\begin{array}{l}\text { آند F4/F3 } \\
\text { آند F3/F4 }\end{array}$ & $\approx$ DLPFC & وسيلكائرى & 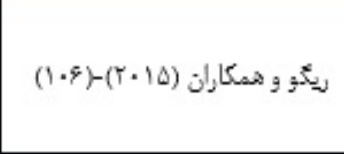 \\
\hline كاهش رقتارهاي يرخاشكرانه & ميلىآمير & . & 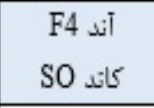 & راست DLPFC & 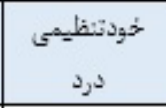 & 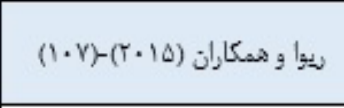 \\
\hline اقزايش هيجانات منفى در & ميلىآمير & ب ب دقيفه & 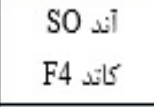 & راست DLPFC & هيجاتات دنفى تنيم & 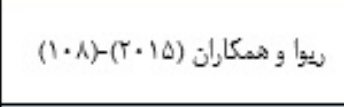 \\
\hline 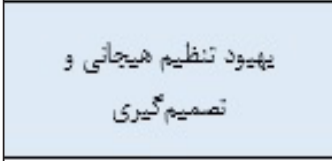 & Tيليآمير & . ب آيقه & 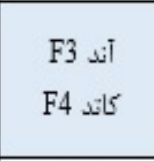 & هLPFC & واكنش هيجانيى & 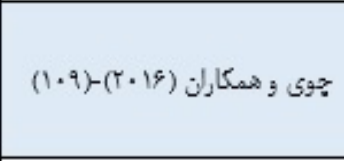 \\
\hline كاهش تكاتشكئرى و إقزايش & ميلىآمير & . ب آقيفه & 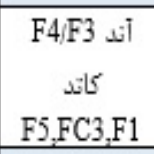 & $\begin{array}{l}\text { DLPFC } \\
\text { צב fاست }\end{array}$ & تون تصميم ميجمانى & ين و همكارلن (T) (T)-ر•) \\
\hline يجيود حاقظل كارى & | اميلي|آمير | & أمئه & 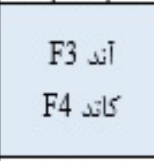 & $\begin{array}{l}\text { DLPFC } \\
\text { צب fاست }\end{array}$ & 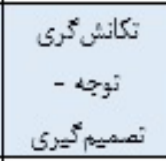 & مانكوسو و همكاران (19 • (T)-(111) \\
\hline 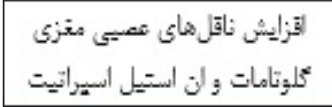 & T بيليآمير & r باتية با. & 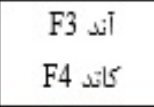 & $\begin{array}{l}\text { DLPFC } \\
\text { הب fاست }\end{array}$ & ماتظد كارى & 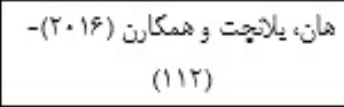 \\
\hline يجيود و تقويت در سويج كردن & ب آميلي آمير & . T بقيفه & 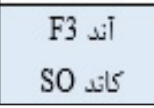 & $\approx$ DLPFC & ناقلين عمييى & تيب و لاويدور (19 - (T)-(T/ا) \\
\hline
\end{tabular}




\begin{tabular}{|c|c|c|c|c|c|c|}
\hline يجيون يادوآورى و اقززايش حاقظله و & هيلمىآمير 1/0 & ه ا قيقيه & 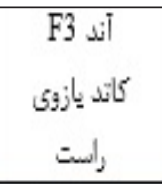 & هLPFC & سولميج كردن & 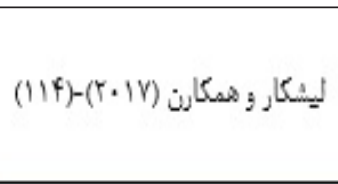 \\
\hline 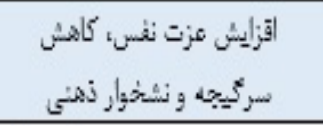 & ميلي آهير & ا انقيفه & 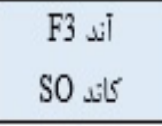 & جLPFC & 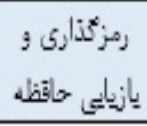 & 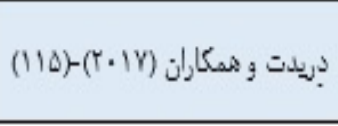 \\
\hline 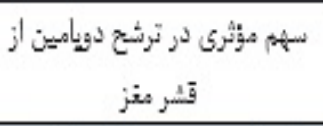 & ب بيليآمير & . تيقيك. & 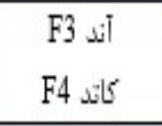 & هLPFC & عزت نفس & 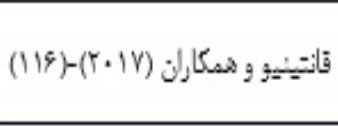 \\
\hline اقززايش نوجه و ماقظة كارى، & $\begin{array}{l}\text { ميلى آمير } 1 / 0 \\
\end{array}$ & . & كاند & جLPFC & ترشيح دويامين & 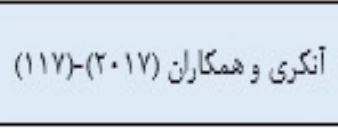 \\
\hline قاقزاليش يهيود در توبها و & ميلي آمير & . & 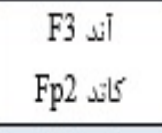 & هي DLPFC & توبه و حاقظة & 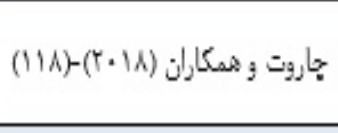 \\
\hline 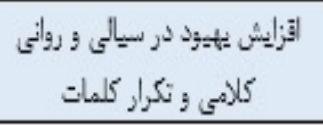 & ا 1 ميليىآمير & . & كاند أند F3 & جLPFC & نوبه & 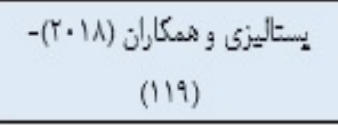 \\
\hline يجيود در تكلم و سيالى كلامى & ب بيليأمير & ه لهيفه & $\begin{array}{l}\text { آند T3/P4F3 } \\
\text { كاتد دلتوئيد }\end{array}$ & هLPFC & آناليز وإزّانان & قناواتى و همكاران (19 • (T)-(1 (19) \\
\hline يليزئد يردازش شناختى و عملكرد & $\begin{array}{c}\text { ميلم آمير } 1 / 0 \\
\end{array}$ & ا 10 اتقيفك & 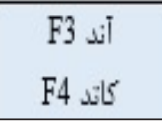 & جLPFC & رواني كادهى & واسفى و ممكارن (19 - (T)-(T) \\
\hline
\end{tabular}

1. Cubelli R, De Bastiani P. 150 years after leborgne: why is paul broca so important in the history of neuropsychology? Cortex. 2011; 2(47): 146-7.

2. Broca P. Sur le siège de la faculté du langage articulé (15 juin). Bulletins de la Société Anthropologque de Paris. 1865; 6: 377-93.

3. Newcombe F. Missile wounds of the brain: a study of psychological deficits. Oxford University Press. 1969.

4. Koenigs M, Tranel D, Damasio AR. The lesion method in cognitive neuroscience. The handbook of psychophysiology. 2007; 139-58.

5. Dayan E, Censor N, Buch ER, Sandrini M, Cohen LG. Noninvasive brain stimulation: from physiology to network dynamics and back. Nat Neurosci. 2013; 16(7): 838-44.

6. Nitsche MA, Liebetanz D, Antal A, Lang N, Tergau F, Paulus W. Modulation of cortical excitability by weak direct current stimulation-technical, safety and functional aspects. Supplements to Clinical Neurophysiology. 2003; 56: 255-76.

7. Boggio PS, Zaghi S, Fregni F. Modulation of emotions associated with images of human pain using anodal transcranial direct current stimulation (tDCS). Neuropsychologia. 2009; 47(1): 212-7.
8. Schlaug G, Renga V, Nair D. Transcranial direct current stimulation in stroke recovery. Archives of Neurology. 2008; 65(12): 1571-6.

9. Gomez Palacio Schjetnan A, Faraji J, Metz GA, Tatsuno M, Luczak A. Transcranial direct current stimulation in stroke rehabilitation: a review of recent advancements. Stroke Research and Treatment. 2013; 2013.

10. Kessler SK, Minhas P, Woods AJ, Rosen A, Gorman C, Bikson M. Dosage considerations for transcranial direct current stimulation in children: a computational modeling study. PloS One. 2013; 8(9): e76112.

11. Kuo MF, Paulus W, Nitsche MA. Therapeutic effects of non-invasive brain stimulation with direct currents (tDCS) in neuropsychiatric diseases. Neuroimage. 2014; 85: $948-60$.

12. Nitsche MA, Boggio PS, Fregni F, Pascual-Leone A. Treatment of depression with transcranial direct current stimulation (tDCS): a review. Experimental Neurology. 2009; 219(1): 14-9.

13. Madhavan S, Shah B. Enhancing motor skill learning with transcranial direct current stimulation-a concise review with applications to stroke. Frontiers in Psychiatry. 2012; 3: 66.

14. Antal A, Kincses TZ, Nitsche MA, Paulus W. 


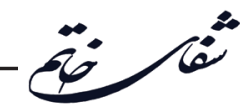

Modulation of moving phosphene thresholds by transcranial direct current stimulation of V1 in human. Neuropsychologia. 2003; 41(13): 1802-7.

15. Flöel A, Rösser N, Michka O, Knecht S, Breitenstein C. Noninvasive brain stimulation improves language learning. Journal of Cognitive Neuroscience. 2008; 20(8): 1415-22.

16. Kincses TZ, Antal A, Nitsche MA, Bártfai O, Paulus W. Facilitation of probabilistic classification learning by transcranial direct current stimulation of the prefrontal cortex in the human. Neuropsychologia. 2004; 42(1): 113-7.

17. Nitsche MA. Transcranial direct current stimulation: a new treatment for depression? Bipolar Disorders. 2002; 4: 98-9.

18. Fregni F, Boggio PS, Mansur CG, Wagner $T$, Ferreira MJ, Lima MC, et al. Transcranial direct current stimulation of the unaffected hemisphere in stroke patients. Neuroreport. 2005; 16(14): 1551-5.

19. Tranulis C, Sepehry AA, Galinowski A, Stip E. Should we treat auditory hallucinations with repetitive transcranial magnetic stimulation? A metaanalysis. The Canadian Journal of Psychiatry. 2008; 53(9): 577-86.

20.BeeliG,CasuttG,BaumgartnerT,JänckeL. Modulating presence and impulsiveness by external stimulation of the brain. Behavioral and Brain Functions. 2008; 4(1): 33.

21. Dockery CA, Hueckel-Weng R, Birbaumer N, Plewnia C. Enhancement of planning ability by transcranial direct current stimulation. Journal of Neuroscience. 2009; 29(22): 7271-7.

22. Leite J, Carvalho S, Fregni F, Boggio PS, Gonçalves ÓF. The effects of cross-hemispheric dorsolateral prefrontal cortex transcranial direct current stimulation (tDCS) on task switching. Brain Stimulation. 2013; 6(4): 660-7.

23. Asthana M, Nueckel K, Mühlberger A, Neueder D, Polak T, Domschke K, et al. Effects of transcranial direct current stimulation on consolidation of fear memory. Frontiers in Psychiatry. 2013; 4: 107.

24. Vannorsdall TD, Schretlen DJ, Andrejczuk M, Ledoux K, Bosley LV, Weaver JR, et al. Altering automatic verbal processes with transcranial direct current stimulation. Frontiers in Psychiatry. 2012; 3: 73.

25. Fecteau S, Knoch D, Fregni F, Sultani N, Boggio P, Pascual-Leone A. Diminishing risk-taking behavior by modulating activity in the prefrontal cortex: a direct current stimulation study. Journal of Neuroscience. 2007; 27(46): 12500-5.

26. Fregni F, Orsati F, Pedrosa W, Fecteau S, Tome FA, Nitsche MA, et al. Transcranial direct current stimulation of the prefrontal cortex modulates the desire for specific foods. Appetite. 2008; 51(1): 34-41.

27. Fregni F, Boggio PS, Nitsche MA, Rigonatti SP, Pascual-Leone A. Cognitive effects of repeated sessions of transcranial direct current stimulation in patients with depression. Depression and Anxiety. 2006; 23(8): 482-4.

28. Boggio PS, Ferrucci R, Rigonatti SP, Covre P, Nitsche M, Pascual-Leone A, et al. Effects of transcranial direct current stimulation on working memory in patients with Parkinson's disease. Journal of the Neurological Sciences. 2006; 249(1): 31-8.

29. Nitsche MA, Cohen LG, Wassermann EM, Priori A, Lang N, Antal A, et al. Transcranial direct current stimulation: state of the art 2008. Brain Stimulation. 2008; 1(3): 206-23.

30. Javadi AH, Walsh V. Transcranial direct current stimulation (tDCS) of the left dorsolateral prefrontal cortex modulates declarative memory. Brain Stimulation. 2012; 5(3): 231-41.

31. Boggio PS, Zaghi S, Lopes M, Fregni F. Modulatory effects of anodal transcranial direct current stimulation on perception and pain thresholds in healthy volunteers. European Journal of Neurology. 2008; 15(10): 1124-30.

32. Teo F, Hoy KE, Daskalakis ZJ, Fitzgerald PB. Investigating the role of current strength in tDCS modulation of working memory performance in healthy controls. Frontiers in Psychiatry. 2011; 2: 45.

33. Tanoue RT, Jones KT, Peterson DJ, Berryhill ME. Differential frontal involvement in shifts of internal and perceptual attention. Brain Stimulation. 2013; 6(4): 675-82.

34. Fregni F, Boggio PS, Nitsche M, Bermpohl F, Antal A, Feredoes E, et al. Anodal transcranial direct current stimulation of prefrontal cortex enhances working memory. Experimental Brain Research. 2006; 166(1): 23-30.

35. Marshall L, Mölle M, Siebner HR, Born J. Bifrontal transcranial direct current stimulation slows reaction time in a working memory task. BMC Neuroscience. 2005; 6(1): 23 .

36. Jacobson L, Koslowsky M, Lavidor M. tDCS polarity 
effects in motor and cognitive domains: a meta-analytical review. Experimental Brain Research. 2012; 216(1) :1-10.

37. Batsikadze G, Moliadze V, Paulus W, Kuo MF, Nitsche MA. Partially non-linear stimulation intensitydependent effects of direct current stimulation on motor cortex excitability in humans. The Journal of Physiology. 2013; 591(7): 1987-2000.

38. Miniussi C, Harris JA, Ruzzoli M. Modelling noninvasive brain stimulation in cognitive neuroscience. Neuroscience \& Biobehavioral Reviews. 2013; 37(8): 1702-12.

39. Silvanto J, Muggleton N, Walsh V. State-dependency in brain stimulation studies of perception and cognition. Trends in Cognitive Sciences. 2008; 12(12): 447-54.

40. Silvanto J, Pascual-Leone A. State-dependency of transcranial magnetic stimulation. Brain Topography. 2008; 21(1): 1 .

41. Quartarone A, Morgante F, Bagnato S, Rizzo V, Sant'Angelo A, Aiello E, et al. Long lasting effects of transcranial direct current stimulation on motor imagery. Neuroreport. 2004; 15(8): 1287-91.

42. Wiethoff S, Hamada M, Rothwell JC. Variability in response to transcranial direct current stimulation of the motor cortex. Brain Stimulation. 2014; 7(3): 468-75.

43. López-Alonso V, Cheeran B, Río-Rodríguez D, Fernández-del-Olmo M. Inter-individual variability in response to non-invasive brain stimulation paradigms. Brain Stimulation. 2014; 7(3): 372-80.

44. Fregni F, Boggio PS, Nitsche MA, Marcolin MA, Rigonatti SP, Pascul-Leone A. Treatment of major depression with transcranial direct current stimulation. Bipolar Disorders. 2006; 8(2): 203-4.

45. Fecteau S, Pascual-Leone A, Zald DH, Liguori P, Théoret H, Boggio PS, et al. Activation of prefrontal cortex by transcranial direct current stimulation reduces appetite for risk during ambiguous decision making. Journal of Neuroscience. 2007; 27(23): 6212-8.

46. Knoch D, Nitsche MA, Fischbacher U, Eisenegger C, Pascual-Leone A, Fehr E. Studying the neurobiology of social interaction with transcranial direct current stimulation-the example of punishing unfairness. Cerebral Cortex. 2007; 18(9): 1987-90.

47. Priori A, Mameli F, Cogiamanian F, Marceglia SA, Tiriticco M, Mrakic-Sposta S, et al. Lie-specific involvement of dorsolateral prefrontal cortex in deception. Cerebral Cortex. 2007; 18(2): 451-5.
48. Beeli G, Koeneke S, Gasser K, Jancke L. Brain stimulation modulates driving behavior. Behavioral and Brain Functions. 2008; 4(1): 34

49. Ohn SH, Park CI, Yoo WK, Ko MH, Choi KP, Kim GM, et al. Time-dependent effect of transcranial direct current stimulation on the enhancement of working memory. Neuroreport. 2008; 19(1): 43-7.

50. Jo JM, Kim YH, Ko MH, Ohn SH, Joen B, Lee $\mathrm{KH}$. Enhancing the working memory of stroke patients using tDCS. American Journal of Physical Medicine \& Rehabilitation. 2009; 88(5): 404-9.

51. Kang EK, Baek MJ, Kim S, Paik NJ. Non-invasive cortical stimulation improves post-stroke attention decline. Restorative Neurology and Neuroscience. 2009; 27(6): $647-52$.

52. Cerruti C, Schlaug G. Anodal transcranial direct current stimulation of the prefrontal cortex enhances complex verbal associative thought. Journal of Cognitive Neuroscience. 2009; 21(10): 1980-7.

53. Elmer S, Burkard M, Renz B, Meyer M, Jäncke L. Direct current induced shortterm modulation of the left dorsolateral prefrontal cortex while learning auditory presented nouns. Behav Brain Funct. 2009; 5: 29.

54. Fertonani A, Rosini S, Cotelli M, Rossini PM, Miniussi C. Naming facilitation induced by transcranial direct current stimulation. Behavioural Brain Research. 2010; 208(2): 311-8.

55. Hecht D, Walsh V, Lavidor M. Transcranial direct current stimulation facilitates decision making in a probabilistic guessing task. Journal of Neuroscience. 2010; 30(12): 4241-5

56. Mameli F, Mrakic-Sposta S, Vergari M, Fumagalli M, Macis M, Ferrucci R, et al. Dorsolateral prefrontal cortex specifically processes general-but not personalknowledge deception: multiple brain networks for lying. Behavioural Brain Research. 2010; 211(2): 164-8.

57. Boggio PS, Campanhã C, Valasek CA, Fecteau S, Pascual-Leone A, Fregni F. Modulation of decisionmaking in a gambling task in older adults with transcranial direct current stimulation. European Journal of Neuroscience. 2010; 31(3): 593-7.

58. Ambrus GZG, Zimmer M, Kincses ZT, Harza I, Kovács G, Paulus W, et al. The enhancement of cortical excitability over the DLPFC before and during training impairs categorization in the prototype distortion task.

Neuropsychologia. 2011; 49(7): 1974-80.59. Andrews 
SC, Hoy KE, Enticott PG, Daskalakis ZJ, Fitzgerald PB. Improving working memory: the effect of combining cognitive activity and anodal transcranial direct current stimulation to the left dorsolateral prefrontal cortex. Brain Stimulation. 2011; 4(2): 84-9.

60. Peña-Gómez C, Vidal-Pineiro D, Clemente IC, Pascual-Leone Á, Bartres-Faz D. Down-regulation of negative emotional processing by transcranial direct current stimulation: effects of personality characteristics. PloS One. 2011; 6(7): e22812.

61. Hammer A, Mohammadi B, Schmicker M, Saliger S, Münte TF. Errorless and errorful learning modulated by transcranial direct current stimulation. BMC Neuroscience. 2011; 12(1): 72.

62. Leite J, Carvalho S, Fregni F, Gonçalves OF. Taskspecific effects of tDCS-induced cortical excitability changes on cognitive and motor sequence set shifting performance. PloS One. 2011; 6(9): e24140.

63. Mulquiney PG, Hoy KE, Daskalakis ZJ, Fitzgerald $\mathrm{PB}$. Improving working memory: exploring the effect of transcranial random noise stimulation and transcranial direct current stimulation on the dorsolateral prefrontal cortex. Clinical Neurophysiology. 2011; 122(12): 2384-9.

64. Peña-Gómez C, Vidal-Pineiro D, Clemente IC, Pascual-Leone Á, Bartres-Faz D. Down-regulation of negative emotional processing by transcranial direct current stimulation: effects of personality characteristics. PloS One. 2011; 6(7): e22812.

65. Zaehle T, Sandmann P, Thorne JD, Jäncke L, Herrmann CS. Transcranial direct current stimulation of the prefrontal cortex modulates working memory performance: combined behavioural and electrophysiological evidence. BMC Neuroscience. 2011; 12(1): 2.

66. Minati L, Campanhã C, Critchley HD, Boggio PS. Effects of transcranial direct-current stimulation (tDCS) of the dorsolateral prefrontal cortex (DLPFC) during a mixed-gambling risky decision-making task. Cognitive Neuroscience. 2012; 3(2): 80-8.

67. Balconi M, Vitaloni S. The tDCS effect on alpha brain oscillation for correct vs. incorrect object use. The contribution of the left DLPFC. Neuroscience Letters. 2012; 517(1): 25-9.

68. Gladwin TE, den Uyl TE, Wiers RW. Anodal tDCS of dorsolateral prefontal cortex during an Implicit Association Test. Neuroscience Letters. 2012; 517(2): 82-6.

69. Hortensius R, Schutter DJ, Harmon-Jones E. When anger leads to aggression: Induction of relative left frontal cortical activity with transcranial direct current stimulation increases the anger-aggression relationship. Social Cognitive and Affective Neuroscience. 2011; 7(3): 342-7.

70. Javadi AH, Cheng P, Walsh V. Short duration transcranial direct current stimulation (tDCS) modulates verbal memory. Brain Stimulation. 2012; 5(4): 468-74.

71. Jeon SY, Han SJ. Improvement of the working memory and naming by transcranial direct current stimulation. Ann Rehabil Med. 2012; 36(5): 585-95.

72. Maeoka H, Matsuo A, Hiyamizu M, Morioka S, Ando $\mathrm{H}$. Influence of transcranial direct current stimulation of the dorsolateral prefrontal cortex on pain related emotions: a study using electroencephalographic power spectrum analysis. Neuroscience Letters. 2012; 512(1): 12-6.

73. Metuki N, Sela T, Lavidor M. Enhancing cognitive control components of insight problems solving by anodal tDCS of the left dorsolateral prefrontal cortex. Brain Stimulation. 2012; 5(2): 110-5.

74. Mylius V, Jung M, Menzler K, Haag A, Khader PH, Oertel WH, et al. Effects of transcranial direct current stimulation on pain perception and working memory. European Journal of Pain. 2012; 16(7): 974-82.

75. Nitsche MA, Koschack J, Pohlers H, Hullemann S, Paulus W, Happe S. Effects of frontal transcranial direct current stimulation on emotional state and processing in healthy humans. Frontiers in Psychiatry. 2012; 3: 58.

76. Sela T, Ivry RB, Lavidor M. Prefrontal control during a semantic decision task that involves idiom comprehension: a transcranial direct current stimulation study. Neuropsychologia. 2012; 50(9): 2271-80.

77. Xue G, Juan CH, Chang CF, Lu ZL, Dong Q. Lateral prefrontal cortex contributes to maladaptive decisions. Proceedings of the National Academy of Sciences. 2012; 109(12): 4401-6.

78. Pripfl J, Neumann R, Köhler U, Lamm C. Effects of transcranial direct current stimulation on risky decision making are mediated by 'hot'and 'cold'decisions, personality, and hemisphere. European Journal of Neuroscience. 2013; 38(12): 3778-85.

79. Cattaneo Z, Lega C, Flexas A, Nadal M, Munar E, Cela-Conde CJ. The world can look better: enhancing beauty experience with brain stimulation. Social Cognitive and Affective Neuroscience. 2013; 9(11): 1713-21. 
80. Fecteau S, Boggio PS, Fregni F, Pascual-Leone A. Modulation of untruthful responses with non-invasive brain stimulation. Frontiers in Psychiatry. 2013; 3: 97.

81. Hecht D, Walsh V, Lavidor M. Bi-frontal direct current stimulation affects delay discounting choices. Cognitive Neuroscience. 2013; 4(1): 7-11.

82. Feeser M, Prehn K, Kazzer P, Mungee A, Bajbouj M. Transcranial direct current stimulation enhances cognitive control during emotion regulation. Brain Stimulation. 2014; 7(1): 105-12.

83. Foerster Á, Rocha S, Wiesiolek C, Chagas AP, Machado G, Silva E, et al. Site-specific effects of mental practice combined with transcranial direct current stimulation on motor learning. European Journal of Neuroscience. 2013; 37(5): 786-94.

84. Hoy KE, Emonson MR, Arnold SL, Thomson RH, Daskalakis ZJ, Fitzgerald PB. Testing the limits: investigating the effect of tDCS dose on working memory enhancement in healthy controls. Neuropsychologia. 2013; 51(9): 1777-84.

85. Javadi AH, Cheng P. Transcranial direct current s timulation (tDCS) enhances reconsolidation of longterm memory. Brain Stimulation. 2013; 6(4): 668-74.

86. Kongthong N, Minami T, Nakauchi S. Semantic processing in subliminal face stimuli: an EEG and tDCS study. Neuroscience Letters. 2013; 544: 141-6.

87. Manenti R, Brambilla M, Petesi M, Ferrari C, Cotelli M. Enhancing verbal episodic memory in older and young subjects after non-invasive brain stimulation. Frontiers in Aging Neuroscience. 2013; 5: 49.

88. Meiron O, Lavidor M. Unilateral prefrontal direct current stimulation effects are modulated by working memory load and gender. Brain Stimulation. 2013; 6(3): 440-7.

89. Mengarelli F, Spoglianti S, Avenanti A, Di Pellegrino G. Cathodal tDCS over the left prefrontal cortex diminishes choice-induced preference change. Cerebral Cortex. 2013; 25(5): 1219-27.

90. Motohashi N, Yamaguchi M, Fujii T, Kitahara Y. Mood and cognitive function following repeated transcranial direct current stimulation in healthy volunteers: a preliminary report. Neuroscience Research. 2013; 77(1-2): 64-9.

91. Nozari N, Thompson-Schill SL. More attention when speaking: Does it help or does it hurt? Neuropsychologia. 2013; 51(13): 2770-80.
92. Vanderhasselt MA, Brunoni AR, Loeys T, Boggio PS, De Raedt R. Nosce te ipsum-Socrates revisited? Controlling momentary ruminative self-referent thoughts by neuromodulation of emotional working memory. Neuropsychologia. 2013; 51(13): 2581-9.

93. Vanderhasselt MA, De Raedt R, Brunoni AR, Campanhã C, Baeken C, Remue J, et al. tDCS over the left prefrontal cortex enhances cognitive control for positive affective stimuli. PloS One. 2013; 8(5): e62219.

94. Balconi M, Vitaloni S. Dorsolateral $\mathrm{pFC}$ and the representation of the incorrect use of an object: the transcranial direct current stimulation effect on N400 for visual and linguistic stimuli. Journal of Cognitive Neuroscience. 2014; 26(2): 305-18.

95. Nelson JT, McKinley RA, Golob EJ, Warm JS, Parasuraman R. Enhancing vigilance in operators with prefrontal cortex transcranial direct current stimulation (tDCS). Neuroimage. 2014; 85: 909-17.

96. Smittenaar P, Prichard G, FitzGerald TH, Diedrichsen J, Dolan RJ. Transcranial direct current stimulation of right dorsolateral prefrontal cortex does not affect modelbased or model-free reinforcement learning in humans. PloS One. 2014; 9(1): e86850.

97. Woods AJ, Hamilton RH, Kranjec A, Minhaus P, Bikson M, Yu J, et al. Space, time, and causality in the human brain. Neuroimage. 2014; 92: 285-97.

98. Feeser M, Prehn K, Kazzer P, Mungee A, Bajbouj M. Transcranial direct current stimulation enhances cognitive control during emotion regulation. Brain Stimulation. 2014; 7(1): 105-12.

99. Sanders T, Liu Y, Buchner V, Tchounwou PB. Neurotoxic effects and biomarkers of lead exposure: a review. Reviews on Environmental Health. 2009; 24(1): $15-46$.

100. Carvalho S, Boggio PS, Gonçalves ÓF, Vigário AR, Faria M, Silva S, et al. Transcranial direct current stimulation based metaplasticity protocols in working memory. Brain Stimulation. 2015; 8(2): 289-94.

101. Kuehne M, Heimrath K, Heinze HJ, Zaehle T. Transcranial direct current stimulation of the left dorsolateral prefrontal cortex shifts preference of moral judgments. PloS One. 2015; 10(5): e0127061.

102. Pripfl J, Lamm C. Focused transcranial direct current stimulation (tDCS) over the dorsolateral prefrontal cortex modulates specific domains of self-regulation. Neurosci Res. 2015; 91: 41-7.

103. Cheng GL, Lee TM. Altering risky decision-making: 


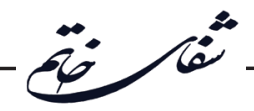

Influence of impulsivity on the neuromodulation of prefrontal cortex. Social Neuroscience. 2016; 11(4): 353-64.

104. Leenus DJF, Cuypers K, Vanvlijmen D, Meesen RLJ. The effect of anodal transcranial direct current stimulation on multi-limb coordination performance. Neuroscience. 2015; 290: 11-7.

105. Turi Z, Mittner M, Opitz A, Popkes M, Paulus W, Antal A. Transcranial direct current stimulation over the left prefrontal cortex increases randomness of choice in instrumental learning. Cortex. 2015; 63: 145-54.

106. Rêgo GG, Lapenta OM, Marques LM, Costa TL, Leite J, Carvalho S, et al. Hemispheric dorsolateral prefrontal cortex lateralization in the regulation of empathy for pain. Neuroscience Letters. 2015; 594: 12-6.

107. Riva P, Romero Lauro LJ, DeWall CN, Chester DS, Bushman BJ. Reducing aggressive responses to social exclusion using transcranial direct current stimulation. Social Cognitive and Affective Neuroscience. 2014; 10(3): 352-6.

108. Riva P, Romero Lauro LJ, Vergallito A, DeWall CN, Bushman BJ. Electrified emotions: Modulatory effects of transcranial direct stimulation on negative emotional reactions to social exclusion. Social Neuroscience. 2015; 10(1): 46-54.

109. Choi KM, Scott DT, Lim SL. The modulating effects of brain stimulation on emotion regulation and decision-making. Neuropsychiatric Electrophysiology. 2016; 2(1): 4 .

110. Ye H, Chen S, Huang D, Wang S, Jia Y, Luo J. Transcranial direct current stimulation over prefrontal cortex diminishes degree of risk aversion. Neuroscience Letters. 2015; 598: 18-22.

111. Mancuso LE, Ilieva IP, Hamilton RH, Farah MJ. Does transcranial direct current stimulation improve healthy working memory? a meta-analytic review. Journal of Cognitive Neuroscience. 2016; 28(8): 1063-89.

112. Hone-Blanchet A, Edden RA, Fecteau S. Online effects of transcranial direct current stimulation in real time on human prefrontal and striatal metabolites. Biological Psychiatry. 2016; 80(6): 432-8.

113. Tayeb Y, Lavidor M. Enhancing switching abilities:
Improving practice effect by stimulating the dorsolateral pre frontal cortex. Neuroscience. 2016; 313: 92-8.

114. Leshikar ED, Leach RC, McCurdy MP, Trumbo MC, Sklenar AM, Frankenstein AN, etv al. Transcranial direct current stimulation of dorsolateral prefrontal cortex during encoding improves recall but not recognition memory. Neuropsychologia. 2017; 106: 390-7.

115. De Raedt R, Remue J, Loeys T, Hooley JM, Baeken C. The effect of transcranial direct current stimulation of the prefrontal cortex on implicit self-esteem is mediated by rumination after criticism. Behaviour Research and Therapy. 2017; 99: 138-46.

116. Fonteneau C, Haesebaert F, Redoute J, Le Bars D, Costes N, Brunelin J, et al. Online transcranial direct current stimulation of the frontal cortex induces dopamine release in the striatum-a spatial and temporal analysis in healthy humans. Brain Stimulation: Basic, Translational, and Clinical Research in Neuromodulation. 2017; 10(2): 516-7.

117. Ankri Y, Meiron O, Braw Y. Executive attention enhancement under stress: A TDCS randomized controlled trial. Brain Stimulation: Basic, Translational, and Clinical Research in Neuromodulation. 2017; 10(2): 350 .

118. Charvet L, Shaw M, Dobbs B, Frontario A, Sherman $\mathrm{K}$, Bikson M, et al. Remotely supervised transcranial direct current stimulation increases the benefit of at-home cognitive training in multiple sclerosis. Neuromodulation: Technology at the Neural Interface. 2018; 21(4): 383-9.

119. Pestalozzi MI, Di Pietro M, Martins Gaytanidis C, Spierer L, Schnider A, Chouiter L, et al. Effects of prefrontal transcranial direct current stimulation on lexical access in chronic poststroke aphasia. Neurorehabilitation and Neural Repair. 2018; 32(10): 913-23.

120. Ghanavati E, Salehinejad MA, Nejati V, Nitsche MA. Differential role of prefrontal, temporal and parietal cortices in verbal and figural fluency: Implications for the supramodal contribution of executive functions. Scientific Reports. 2019; 9(1): 3700.

121. Vaseghi B, Zoghi M, Jaberzadeh S. Unihemispheric concurrent dual-site cathodal transcranial direct current stimulation: the effects on corticospinal excitability. European Journal of Neuroscience. 2016; 43(9): 1161-72. 